\title{
Leptonic CP violating effective action for Dirac and Majorana neutrinos
}

\author{
Carmen García-Recio and Lorenzo Luis Salcedo \\ Departamento de Física Atómica, Molecular y Nuclear and \\ Instituto Carlos I de Física Teórica y Computacional, \\ Universidad de Granada, E-18071 Granada, Spain \\ E-mail: g_recio@ugr.es, salcedo@ugr.es
}

ABSTRACT: In the Standard Model minimally extended to include massive neutrinos, we compute the leading CP-violating zero temperature contributions to the one-loop effective action induced by integration of the leptons. Such contributions start at operators of dimension six and they are $\mathrm{P}$ even for Dirac neutrinos and $\mathrm{P}$ even or odd for Majorana neutrinos. Dimension four operators are allowed in the mixed Dirac-Majorana case. It is verified by explicit calculation that $\mathrm{CP}$ can be violated in two generation settings for Majorana neutrinos. Using different neutrino scenarios we give upper bounds for the couplings of the CP-violating operators. As a rule, we find that lepton-induced couplings are suppressed as compared to quark-induced couplings, whenever the latter are allowed, nevertheless, through virtual lepton-number violating mechanisms, Majorana neutrinos induce new CP-violating operators not present in the quark or Dirac-neutrino cases.

Keywords: CP violation, Neutrino Physics

ARXIV EPRINT: 1405.7927 


\section{Contents}

1 Introduction $\quad 1$

2 Dirac and Majorana chiral gauge fermions 5

2.1 Dirac fermions 5

2.2 Majorana fermions 9

3 The (extended) Standard Model $\quad 11$

$\begin{array}{lll}3.1 & \text { Leptonic sector of the Standard Model } & 11\end{array}$

$\begin{array}{lll}3.2 & \text { Neutrinos with mixed Dirac-Majorana mass terms } & 12\end{array}$

4 CP odd component of the effective action $\quad 14$

$\begin{array}{lll}5 & \text { Operator } \mathrm{K} \text { for Majorana neutrinos } & 17\end{array}$

6 Effective action in the CP odd sector $\quad 22$

$6.1 \Gamma_{4}$ for Majorana and Dirac neutrinos 22

6.2 Method of covariant symbols 24

6.3 Allowed operators and their couplings 25

$\begin{array}{ll}6.4 \text { Discussion of the analytical results } & 29\end{array}$

$\begin{array}{lll}7 & \text { Invariants and couplings } & 31\end{array}$

$\begin{array}{lll}7.1 & \text { Invariants } & 31\end{array}$

7.2 Couplings of Dirac type 33

$\begin{array}{lll}7.3 & \text { Couplings of Majorana type } & 35\end{array}$

$\begin{array}{lll}7.4 & \text { Leptons vs quarks and numerical estimates } & 37\end{array}$

8 Summary and conclusions $\quad 40$

$\begin{array}{ll}\text { A Covariant and ordinary symbols } & 41\end{array}$

\section{Introduction}

Neutrino physics is nowadays quite an active field of research, from several directions. These include nuclear physics (neutrinoless double beta decay, matter effects, response functions of weak currents in nuclei), particle physics (neutrino detection, neutrino oscillations, Standard Model extensions, Pontecorvo-Maki-Nakagawa-Sakata (PMNS) matrix, $\mathrm{CP}$ violation, lepton number violation, sterile neutrinos), astrophysics (neutrino production in stars, supernovae dynamics, neutrino telescopes), cosmological (dark energy, inflation, 
primordial neutrinos), and even more speculative ones such as using neutrinos for communication through quantum channels. More importantly, these are not separated fields, rather they are closely interconnected in such a way that advances in one fields sheds light on all other fields as well [1-14].

On the other hand, CP violation remains as a challenging subject [15-23] since its discovery fifty years ago [24] and subsequent observation of direct CP violation [25, 26]. $\mathrm{CP}$ violation plays a key role in the understanding of baryo- and leptogenesis [27-29], timereversal violation (through CPT invariance) or the electric dipole moments of particles [30]. There is no generally accepted explanation for the non-violation of CP symmetry in the strong interaction sector [31]. In the electroweak sector, CP violation enters through the flavor mixing complex mass matrices of the fermions, the Cabibbo-Kobayashi-Maskawa (CKM) matrix for quarks [32] and the PMNS matrix for leptons [33-35]. The CKM matrix elements are currently known with some precision and the CP-violating phase turns out to be rather small [36]. For the PMNS matrix, the angles are being measured in current experiments [37-40] while no information is currently available for the phases on which CP violation depends.

In the present work, we deal with the effective action of the Standard Model (extended to include neutrino masses) and more concretely with its $\mathrm{CP}$ violating component. By effective action here we refer to the functional obtained by integration of the fermions (quarks and leptons) in the theory. Such functional depends on the configurations of the unintegrated fields in the Standard Model, namely, the gauge bosons ( $W^{ \pm}, Z^{0}$, photon and gluons) and the Higgs field. The effective action so defined, $\Gamma$, is a complicated gauge invariant functional of these bosonic fields. In order to organize this functional we adopt a local expansion, namely, classifying the terms by their number of covariant derivatives,

$$
\Gamma=\int d^{4} x \sum_{i} g_{i} \mathcal{O}_{i}(x)
$$

The quantities $\mathcal{O}_{i}(x)$ stand for the possible local operators (monomials) that can be constructed using the available fields, restricted by gauge and Lorentz covariance, etc. The $g_{i}$ are the couplings of these operators in the effective action of the (extended) Standard Model. Each operator has a certain number of covariant derivatives. In this counting the gauge fields count as one derivative, therefore (barring the Higgs field) the operators are essentially classified by their mass dimension. ${ }^{1}$ We aim at the computation of the couplings to the leading (lowest dimensional) operators which are CP odd. The effective action has been modeled before in the literature, assuming phenomenological values or estimates for the couplings to non-renormalizable operators, with the purpose of studying electroweak baryogenesis or electric dipole moments of particles [41-46]. At variance with this phenomenological approach, our purpose here is to carry out a direct calculation of the couplings using a strict derivative expansion starting from the Standard Model Lagrangian.

\footnotetext{
${ }^{1}$ Of course, the mass dimension carried by the Higgs field is relevant for the (non) renormalizability of the operators. The Higgs field is properly included in our calculation below, we merely disregard it in our classification of operators.
} 
The specific motivation for this calculation comes from the observation by Smit [47] that CP violation needs not be parametrically small in the Standard Model. It is wellknown that CP violation, even if allowed in the Standard Model through the KobayashiMaskawa mechanism, is a rather elusive phenomenon. For quarks or Dirac neutrinos it requires the participation of at least three generations to have a non vanishing result. This is best summarized by the Jarlskog determinant which involves the CKM matrix through a very specific combination, the Jarlskog invariant [48], and the quarks masses also in a very specific combination, $\prod_{i<j=u, c, t}\left(m_{i}^{2}-m_{j}^{2}\right) \prod_{i<j=d, s, b}\left(m_{i}^{2}-m_{j}^{2}\right)$. The Jarlskog determinant is a twelfth degree polynomial in the masses which must be present, as a factor, in any CP violating contribution [49]. If the Jarlskog determinant is simply compensated with the appropriate power of $v$, the Higgs vacuum expectation value, one obtains extremely small ratios: the dimensionless ratio obtained by dividing by $v^{12}$ gives a number as small as $10^{-24}$ for quarks. This is a parametrically small result that comes from assuming a perturbative treatment for the fermion masses. The observation in [47] is that, instead of polynomials one should expect rational functions (plus logarithms) of the fermion masses and this may lead to a substantial increase in the estimate of the strength of the couplings to $\mathrm{CP}$ violating operators at zero temperature (the only case we consider throughout this work).

Calculations along these lines were carried out for quarks and dimension six operators in [50] for the $\mathrm{P}$ odd sector only, and in [51] for the two parity sectors and including also the Higgs field. Unfortunately, the results of the two groups, obtained by two different methods, are mutually incompatible. The result obtained in [51], has been reproduced in [52] using the same method as in [51] and also in [53], this time using the same method as in [50]. The couplings to selected dimension eight operators have been obtained in [53] and [54]. For Dirac particles these are the first instances of $\mathrm{P}$ odd $\mathrm{CP}$ violating contributions. Extensions to finite temperature have been addressed in $[52,54]$.

In those calculations one indeed finds a large enhancement in the value of the couplings, as compared to perturbative estimates. Such larger couplings would have an impact on the viability of cold electroweak baryogenesis scenarios $[52,55,56]$. Ultimately, the enhancement comes from the fact that the typical scale in the coupling is not set by value of the Higgs condensate but rather by the quark masses themselves and some of them are relatively small. ${ }^{2}$ However, the precise combinations of masses are not obvious without a detailed calculation. For dimension six operators, what is actually found is that the coupling comes from a loop momentum integral which would be afflicted by infrared (IR) divergences for massless $u, d$ and $s$ quarks. As a consequence, finite but different results are obtained depending on how the ratios between light quark masses are taken in that massless limit.

The coupling to dimension six CP violating operators just discussed does not have a contribution from leptons in the strict Standard Model, where neutrinos are massless. In fact, the leptonic loop exactly preserves CP symmetry for massless neutrinos. Nevertheless, the scheme used for quark applies quite directly to massive neutrinos of Dirac type. The

\footnotetext{
${ }^{2}$ Equivalently, disregarding the very disparate scales in the values of the Yukawa couplings is not a good enough estimate.
} 
small neutrino masses calls for an investigation of how the possible IR divergencies affect the couplings in the leptonic sector. In some sense the leptonic case is cleaner than the quarkonic one, as gluonic corrections (which start at dimension 8) are not present. On the other hand, the information on neutrino masses and the PMNS matrix is currently less complete than for quarks. In addition, neutrinos may have mass terms of Majorana type that can be accommodated in the Standard Model invoking a seesaw mechanism [57-60]. It is of interest to investigate how the small masses of Majorana type reflect on the couplings to $\mathrm{CP}$ violating operators. This requires a full new determination of the couplings, as the Dirac results can not be directly adapted to describe the Majorana case. In this work we consider these two cases, pure Dirac and pure Majorana neutrinos, with three light flavors although some of the formulas are more general. As we show, the induced CP violating operators have at least dimension six. The mixed case, with mass terms of Dirac and Majorana type simultaneously, is also interesting as it allows dimension four CP odd operators but it is beyond the scope of the present work.

Section 2 reviews aspects of chiral gauge fermions with Dirac mass terms. There we present a new derivation of the technique first introduced in [61] to reduce normal and abnormal parity components of the fermionic effective action to a gauge covariant KleinGordon approach, based on the operator $K$. In the second part of that section we adapt the previous approach to include Majorana mass terms, in addition to the Dirac mass ones, in such a way that the effective action also follows from the determinant of $K$.

In section 3 we spell out how the the previous formalism applies to the leptonic sector of the Standard Model. Before restricting ourselves to the cases of pure Dirac or pure Majorana neutrino masses, in the second part of the section we briefly discuss the general case of mixed Dirac plus Majorana masses.

Section 4 discusses the extraction of the CP odd component of the effective action with an analysis on the types of allowed contributions. There it is shown that also for Majorana neutrinos the leading CP violating terms are of dimension six, with four $W$ fields. However, a new lepton-number violating mechanism is present in the Majorana case, which works even for two generations, in addition to the usual Kobayashi-Maskawa mechanism already present in the Dirac neutrino or quark cases. New mechanisms are found in the mixed Dirac-Majorana case which involves no charged gauge bosons.

The operator $K$ for the Standard Model with Dirac or Majorana neutrinos is constructed in detail in section 5. A direct application of the definition of $K$ in the Majorana case leads to expressions with inverse powers of the neutrino mass matrix, although they dissappear from the final amplitudes. In that section we show how to remove these inverse powers directly from the $K$ operator, from which the effective action follows.

Section 6 describes the explicit computation of the effective action in the CP odd sector, for the lowest dimensional operators. The calculation is based on the technique of covariant symbols $[62,63,79]$ which directly delivers covariant operators in the derivative expansion. In that section the allowed operators are listed together with relations among them from integration by parts, Bianchi identities or transference of Lorentz indices from "metric" type to "exterior algebra" type. Explicit results for the couplings are given in terms of momentum integrals involving masses and the PMNS matrix. Analytical regularities in the results are discussed there. 
Section 7 is devoted to analyzing the results obtained in the previous section. The new invariants that emerge in the Majorana case, in addition to the usual Jarlskog invariant, are identified. Taking advantage of the small neutrino masses, reliable approximate formulas are derived for the couplings of Dirac type and Majorana type. The formulas are particularized for three typical scenarios considered in the literature, namely, quasi degenerate neutrino masses, and normal and inverted hierarchies. Numerical estimates for the couplings to $\mathrm{CP}$ violating operators are given for each of the scenarios. For Dirac neutrinos, it is shown that the different light-heavy patterns in the quark and lepton sectors, as regards to weak isospin, imply a suppression of lepton contributions as compared to quarks contributions. At the same time, for Majorana neutrinos new operators are activated at leading order in the $\mathrm{P}$ violating sector.

Section 8 summarizes our conclusions.

\section{Dirac and Majorana chiral gauge fermions}

\subsection{Dirac fermions}

We start by reviewing Dirac chiral gauge fermions since eventually Majorana fermions will be reduced to this case. We closely follow the exposition in [51] and use the same conventions, so further details can be looked up in that reference. For convenience we work in Euclidean space. The rules to go back and forth between Minkowskian and Euclidean spaces can be found in [51].

For Dirac fermions we consider a generic Lagrangian of the form

$$
\begin{aligned}
\mathcal{L}(x) & =\bar{\psi}(x) \mathbf{D} \psi(x) \\
& =\bar{\psi}_{R} \not D_{R} \psi_{R}+\bar{\psi}_{L} \not_{L} \psi_{L}+\bar{\psi}_{L} m_{L R} \psi_{R}+\bar{\psi}_{R} m_{R L} \psi_{L}
\end{aligned}
$$

where

$$
D_{\alpha}^{L, R}=\partial_{\alpha}+V_{\alpha}^{L, R}
$$

and $V_{\alpha}^{L, R}(x)$ and $m_{L R}(x)$ and $m_{R L}(x)$ are external bosonic fields which are matrices in the internal space of the fermions. (Euclidean) unitarity requires

$$
m_{L R}(x)=m_{R L}^{\dagger}(x), \quad V_{L, R}^{\dagger}(x)=-V_{L, R}(x) .
$$

In the chiral representation of the Dirac gammas, $\psi_{R}$ and $\bar{\psi}_{L}$ have only upper components, and $\psi_{L}$ and $\bar{\psi}_{R}$ have only lower components. The fermionic sector of the Standard Model fits in the scheme of eq. (2.1) when all fermions are of Dirac type [51]. Later below we show that it also can accommodate Majorana fermions.

Integration of the fermionic fields provides the effective action $\Gamma$ :

$$
\begin{aligned}
Z=\int & D \psi_{L} D \psi_{R} D \bar{\psi}_{L} D \bar{\psi}_{R} e^{-\int d^{4} x \mathcal{L}(x)}=\operatorname{Det} \mathbf{D}, \\
\Gamma\left[m_{L R}, m_{R L}, V_{L}, V_{R}\right]=-\log Z & =-\operatorname{Tr} \log \mathbf{D} .
\end{aligned}
$$

This functional just sums all one-loop Feynman graphs with the fermion running on the loop with bosonic external fields attached to it. In this paper by effective action we will 
always mean the one-loop effective action from integration of the fermions, and not the full effective action which would include higher loop graphs with internal gauge and Higgs boson lines.

The effective action is invariant under $\mathrm{CP}$ transformations

$$
\begin{aligned}
m_{L R}(x) & \rightarrow m_{L R}^{*}(\tilde{x}), & m_{R L}(x) & \rightarrow m_{R L}^{*}(\tilde{x}), \\
V_{R, \alpha}(x) & \rightarrow \pi_{\alpha \beta} V_{R, \beta}^{*}(\tilde{x}), & V_{L, \alpha}(x) & \rightarrow \pi_{\alpha \beta} V_{L, \beta}^{*}(\tilde{x}),
\end{aligned}
$$

with $\pi_{\alpha \beta}=\operatorname{diag}(1,-1,-1,-1), \quad \tilde{x}_{\alpha}=\pi_{\alpha \beta} x_{\beta}$.

The effective action can be naturally separated into its parity preserving and parity violating components,

$$
\Gamma=\Gamma^{+}+\Gamma^{-} .
$$

$\Gamma^{-}$and $\Gamma^{+}$are the components with and without the Levi-Civita pseudotensor, respectively. As a consequence of CPT invariance, $\Gamma^{+}$is purely real and $\Gamma^{-}$is purely imaginary, in Euclidean space [64]. Therefore, modulo ultraviolet (UV) ambiguities,

$$
\Gamma^{ \pm}=-\frac{1}{2}\left(\operatorname{Tr} \log \mathbf{D} \pm \operatorname{Tr} \log \mathbf{D}^{\dagger}\right)
$$

The Lagrangian $\mathcal{L}(x)$ is invariant under local chiral transformations. To expose the chiral properties it will prove convenient to write the Lagrangian in matricial form, namely,

$$
\mathcal{L}(x)=\left(\begin{array}{ll}
\bar{\psi}_{L} & \bar{\psi}_{R}
\end{array}\right)\left(\begin{array}{cc}
m_{L R} & \not D_{L} \\
\not D_{R} & m_{R L}
\end{array}\right)\left(\begin{array}{l}
\psi_{R} \\
\psi_{L}
\end{array}\right) .
$$

Chiral gauge transformations take the form

$$
\mathbf{D} \rightarrow \mathbf{D}^{\Omega}=\left(\begin{array}{cc}
\Omega_{L}^{\dagger}(x) & 0 \\
0 & \Omega_{R}^{\dagger}(x)
\end{array}\right) \mathbf{D}\left(\begin{array}{cc}
\Omega_{R}(x) & 0 \\
0 & \Omega_{L}(x)
\end{array}\right)
$$

where $\Omega_{L, R}(x)$ are unitary matrices in internal space.

When $\Omega_{L}=\Omega_{R}$ (vector transformations) $\mathbf{D}$ y $\mathbf{D}^{\Omega}$ are related by a similarity transformation, as a consequence they have the same spectrum and the same effective action. In the general chiral case the two effective actions $\Gamma(\mathbf{D})$ and $\Gamma\left(\mathbf{D}^{\Omega}\right)$ have equal $U V$ convergent contributions, since these are unambiguously fixed by the Lagrangian, but may differ in $U V$ divergent ones. More specifically, from eq. (2.7) it follows that $\Gamma^{+}$can be obtained from the determinant of $\mathbf{D D}^{\dagger}$. This latter operator transforms under a similarity transformation,

$$
\mathbf{D D}^{\dagger} \rightarrow\left(\begin{array}{cc}
\Omega_{L}^{\dagger}(x) & \\
& \Omega_{R}^{\dagger}(x)
\end{array}\right) \mathbf{D D}^{\dagger}\left(\begin{array}{lll}
\Omega_{L}(x) & \\
& & \Omega_{R}(x)
\end{array}\right),
$$

and so it can be regularized in a chirally invariant manner. On the contrary $\Gamma^{-}$has a chiral variation, the chiral anomaly, which cannot be consistently removed [65-67]. The chiral anomaly is saturated by the gauged Wess-Zumino-Witten action (WZW) action [68, 69], and the remaining terms in $\Gamma^{-}$are chirally invariant. Denoting by $\Gamma_{c}$ the chirally invariant component of $\Gamma$, one has

$$
\Gamma^{+}=\Gamma_{c}^{+}, \quad \Gamma^{-}=\Gamma_{c}^{-}+\Gamma_{\mathrm{gWZW}}
$$


The anomalous gauged WZW is known in closed-form and, as we will argue below, it gives no contribution to $\mathrm{CP}$ violation, with either Dirac or Majorana neutrinos.

The chiral invariant reminder $\Gamma_{c}[m, V]$ is a functional of the external fields that admits no closed-form in general. Therefore expansions, such as a the derivative expansion, must be adopted. Nevertheless the chiral invariance of $\Gamma_{c}$ implies a large simplification in the calculations since everything can be expressed in terms of $m_{L R}$ and $m_{R L}$, their chiral covariant derivatives, and the field strengths,

$$
\begin{aligned}
\hat{D}_{\alpha} m_{L R} & =D_{\alpha}^{L} m_{L R}-m_{L R} D_{\alpha}^{R}, \quad \hat{D}_{\alpha} m_{R L}=D_{\alpha}^{R} m_{R L}-m_{R L} D_{\alpha}^{L}, \\
F_{\alpha \beta}^{L, R} & =\left[D_{\alpha}^{L, R}, D_{\beta}^{L, R}\right] .
\end{aligned}
$$

A complete calculation of $\Gamma_{c}$ to four covariant derivatives can be found in [70-72]. However, the calculation gets very involved for higher orders. As we argue below, the sixth order is needed in the derivative expansion to pick up the leading CP violating terms of the effective action. ${ }^{3}$ To obtain those we follow [51] and take the approach of [61], although here we present an alternative derivation.

Consider the well-known relation [73]

$$
\int d^{n} \psi d^{n} \bar{\psi} e^{-\bar{\psi} M \psi+\bar{\eta} \psi+\bar{\psi} \eta}=\operatorname{det} M e^{\bar{\eta} M^{-1} \eta}
$$

and separate the Grassman variables in two types

$$
\bar{\psi} M \psi=\left(\begin{array}{ll}
\bar{\psi}_{1} & \bar{\psi}_{2}
\end{array}\right)\left(\begin{array}{ll}
M_{1,1} & M_{1,2} \\
M_{2,1} & M_{2,2}
\end{array}\right)\left(\begin{array}{l}
\psi_{1} \\
\psi_{2}
\end{array}\right)
$$

where the $M_{i, j}$ are themselves matrices in general. By integrating first $\psi_{1}$ and $\bar{\psi}_{1}$, and then $\psi_{2}$ and $\bar{\psi}_{2}$, or the other way around, the following identities are obtained

$$
\begin{aligned}
\operatorname{det} M & =\operatorname{det} M_{2,2} \operatorname{det}\left(M_{1,1}-M_{1,2} M_{2,2}^{-1} M_{2,1}\right) \\
& =\operatorname{det} M_{1,1} \operatorname{det}\left(M_{2,2}-M_{2,1} M_{1,1}^{-1} M_{1,2}\right) .
\end{aligned}
$$

These identities can be applied directly to the chiral fermions in eq. (2.8):

$$
\operatorname{Det} \mathbf{D}=\operatorname{Det}\left(\kappa_{L}\right)=\operatorname{Det}\left(\bar{\kappa}_{R}\right)
$$

with $^{4}$

$$
\begin{aligned}
& \kappa_{L}=m_{L R} m_{R L}-\sigma_{\alpha} D_{L \alpha} m_{R L}^{-1} \bar{\sigma}_{\beta} D_{R \beta} m_{R L}, \\
& \bar{\kappa}_{R}=m_{R L} m_{L R}-\bar{\sigma}_{\alpha} D_{R \alpha} m_{L R}^{-1} \sigma_{\beta} D_{L \beta} m_{L R} .
\end{aligned}
$$

Here, $\sigma_{\alpha}$ and $\bar{\sigma}_{\alpha}$ are the Pauli and identity matrices corresponding to the chiral representation of the the Dirac gammas:

$$
\gamma_{\alpha}=\left(\begin{array}{cc}
0 & \sigma_{\alpha} \\
\bar{\sigma}_{\alpha} & 0
\end{array}\right), \quad \gamma_{5}=\left(\begin{array}{cc}
1 & 0 \\
0 & -1
\end{array}\right) .
$$

\footnotetext{
${ }^{3}$ At least for pure Dirac or Majorana neutrinos (see eq. (4.11)).

${ }^{4}$ Here we are assuming that $m_{L R}$ is a square and regular matrix. This is natural in parity preserving theories but not in chiral theories. Nevertheless this case is sufficiently general for our purposes.
} 
To avoid working with Dirac bispinors, we introduce the operators

$$
\begin{aligned}
& K_{L}=m_{L R} m_{R L}-\not D_{L} m_{R L}^{-1} \not D_{R} m_{R L}=\left(\begin{array}{cc}
\kappa_{L} & 0 \\
0 & \bar{\kappa}_{L}
\end{array}\right), \\
& K_{R}=m_{R L} m_{L R}-\not D_{R} m_{L R}^{-1} \not D_{L} m_{L R}=\left(\begin{array}{cc}
\kappa_{R} & 0 \\
0 & \bar{\kappa}_{R}
\end{array}\right) .
\end{aligned}
$$

These two operators are related through the identity

$$
K_{R}=m_{L R}^{-1} K_{L}^{\dagger} m_{L R}
$$

In terms of these operators

$$
\begin{aligned}
\operatorname{Tr}\left(\log \kappa_{L}\right) & =\operatorname{Tr}\left(P_{R} \log K_{L}\right), \quad \operatorname{Tr}\left(\log \bar{\kappa}_{R}\right)=\operatorname{Tr}\left(P_{L} \log K_{R}\right), \\
P_{L, R} & =\frac{1}{2}\left(1 \mp \gamma_{5}\right),
\end{aligned}
$$

and we finally obtain

$$
\begin{aligned}
\Gamma_{c} & =-\operatorname{Tr}\left(P_{R} \log K_{L}\right)=-\operatorname{Tr}\left(P_{L} \log K_{R}\right), \\
\Gamma_{c}^{+} & =-\frac{1}{2} \operatorname{Tr}\left(\log K_{L}\right)=-\frac{1}{2} \operatorname{Tr}\left(\log K_{R}\right), \\
\Gamma_{c}^{-} & =-\frac{1}{2} \operatorname{Tr}\left(\gamma_{5} \log K_{L}\right)=+\frac{1}{2} \operatorname{Tr}\left(\gamma_{5} \log K_{R}\right) .
\end{aligned}
$$

To make the identifications with $\Gamma_{c}^{+}$and $\Gamma_{c}^{-}$above, we have used that only $\gamma_{5}$ introduces the Levi-Civita pseudotensor after taking Dirac traces. On the other hand the identity (2.20) implies that $\Gamma_{c}^{+}$is purely real and $\Gamma_{c}^{-}$is purely imaginary (in Euclidean space).

The usefulness of the relations (2.22) is two-fold, first, $K_{L, R}$ are operators of KleinGordon type. While this was already the case for $\mathbf{D D}^{\dagger}$, to obtain $\Gamma_{c}^{+}$, no such operator was available for $\Gamma_{c}^{-}$before [61]. This allows a substantial simplification of the Dirac algebra in the calculations. And second, $K_{L, R}$ are chiral covariant, that is,

$$
K_{L} \rightarrow \Omega_{L}^{\dagger} K_{L} \Omega_{L}, \quad K_{R} \rightarrow \Omega_{R}^{\dagger} K_{R} \Omega_{R}
$$

This fact guarantees that explicit chiral gauge invariance can be maintained throughout the calculation, also in the parity odd component of the effective action (the component afflicted by chiral anomalies).

It is also of interest to see the relation of the operators $K_{L, R}$ with an effective Lagrangian of the Klein-Gordon type for the fermionic amplitudes. The propagator is given by

$$
\left\langle\left(\begin{array}{l}
\psi_{R}(x) \\
\psi_{L}(x)
\end{array}\right)\left(\bar{\psi}_{L}\left(x^{\prime}\right) \bar{\psi}_{R}\left(x^{\prime}\right)\right)\right\rangle=\left\langle x\left|\mathbf{D}^{-1}\right| x^{\prime}\right\rangle .
$$

On the other hand, the inverse Dirac operator can be written as

$$
\mathbf{D}^{-1}=\left(\begin{array}{cc}
\left(m_{L R}-\not_{L} m_{R L}^{-1} \not D_{R}\right)^{-1} & \left(\not D_{R}-m_{R L} \not D_{L}^{-1} m_{L R}\right)^{-1} \\
\left(\not D_{L}-m_{L R} \not D_{R}^{-1} m_{R L}\right)^{-1} & \left(m_{R L}-\not D_{R} m_{L R}^{-1} \not D_{L}\right)^{-1}
\end{array}\right) .
$$


Comparing with the definitions of $K_{L, R}$ in eq. (2.19), it follows that

$$
\left\langle x\left|P_{R} K_{L}^{-1}\right| x^{\prime}\right\rangle=m_{R L}^{-1}\left\langle\psi_{R}(x) \bar{\psi}_{L}\left(x^{\prime}\right)\right\rangle, \quad\left\langle x\left|P_{L} K_{R}^{-1}\right| x^{\prime}\right\rangle=m_{L R}^{-1}\left\langle\psi_{L}(x) \bar{\psi}_{R}\left(x^{\prime}\right)\right\rangle .
$$

Therefore the effective Lagrangian $\bar{\psi}_{L} K_{L} \tilde{\psi}_{R}(x)$, with $\tilde{\psi}_{R} \equiv m_{R L}^{-1} \psi_{R}$, correctly describes the propagator $\left\langle\psi_{R} \bar{\psi}_{L}\right\rangle$, although it gives no direct information on, e.g., $\left\langle\psi_{L} \bar{\psi}_{L}\right\rangle$, and similarly for $K_{R}$.

The manipulations leading to (2.15) and (2.22) are based on the formal identity

$$
\operatorname{Tr} \log (A B)=\operatorname{Tr} \log (A)+\operatorname{Tr} \log (B),
$$

where $A$ and $B$ are differential or pseudodifferential operators. In fact, the determinant of these operators contains UV divergences which have to be removed by means of some renormalization procedure (e.g., $\zeta$-function [74-76]). The choice of renormalization introduces finite ambiguities which can give corrections to the formal identity. This is the origin of the quantum anomalies and the gauged WZW term above. (For a careful treatment including everything as in $(2.11)$ see $[61,71,77]$.) On the other hand, the formal identity holds for the UV finite contributions. Within the derivative expansion, the UV convergent terms are those of order six and higher which we will compute below for the CP odd sector. Therefore no quantum ambiguities nor anomalies appear in the CP violating sector.

\subsection{Majorana fermions}

The fermion Lagrangian including Majorana mass terms is of the form ${ }^{5}$

$$
\begin{aligned}
\mathcal{L}(x)= & \bar{\psi}_{R} \not D_{R} \psi_{R}+\bar{\psi}_{L} \not D_{L} \psi_{L}+\bar{\psi}_{L} m_{L R} \psi_{R}+\bar{\psi}_{R} m_{R L} \psi_{L} \\
& +\frac{1}{2} \bar{\psi}_{L}^{c} m_{L} \psi_{L}+\frac{1}{2} \bar{\psi}_{L} m_{L}^{\dagger} \psi_{L}^{c}+\frac{1}{2} \bar{\psi}_{R}^{c} m_{R} \psi_{R}+\frac{1}{2} \bar{\psi}_{R} m_{R}^{\dagger} \psi_{R}^{c}
\end{aligned}
$$

Here, as usual, $\psi_{L, R}^{c} \equiv C \bar{\psi}_{L, R}^{T}$ and $\bar{\psi}_{L, R}^{c} \equiv-\psi_{L, R}^{T} C^{\dagger}, C$ being the unitary matrix such that $C^{\dagger} \gamma_{\alpha} C=-\gamma_{\alpha}^{T}$. The Majorana mass complex matrices $m_{L}(x)$ and $m_{R}(x)$ are symmetric since $C$ is antisymmetric. Under $\mathrm{CP}$ and chiral gauge transformations they transform, respectively, as

$$
\begin{aligned}
& m_{L}(x) \rightarrow m_{L}^{\dagger}(\tilde{x}), \quad m_{R}(x) \rightarrow m_{R}^{\dagger}(\tilde{x}) \\
& m_{L}(x) \rightarrow \Omega_{L}^{T}(x) m_{L}(x) \Omega_{L}(x), \quad m_{R}(x) \rightarrow \Omega_{R}^{T}(x) m_{R}(x) \Omega_{R}(x) .
\end{aligned}
$$

In the Euclidean formulation, $\psi_{L, R}(x)$ and $\bar{\psi}_{L, R}(x)$ are independent fields to be integrated over. On the other hand, $\psi_{L, R}^{c}, \bar{\psi}_{L, R}^{c}$ are merely auxiliary variables $\left(\bar{\psi}_{L}^{c}\right.$ has the same content as $\psi_{L}$, etc). The Lagrangian with Dirac and Majorana mass terms can be

\footnotetext{
${ }^{5}$ Further "Majorana" vector couplings can be considered, of the type $\frac{1}{2} \bar{\psi}_{L}^{c} A_{L R} \psi_{R}+\frac{1}{2} \bar{\psi}_{R}^{c} A_{R L} \psi_{L}+$ H.c., however, such terms are not present in the Standard Model or any renormalizable theory. Renormalizability would require these vector fields to be of gauge type and the gauge group would mix different chiralities which is forbidden for an internal symmetry.
} 
written in matrix form using the trick of duplicating the size of the matrices, specifically (cf. eq. (2.8))

$$
\mathcal{L}(x)=\frac{1}{2}\left(\psi_{R}^{T} \psi_{L}^{T} \bar{\psi}_{R} \bar{\psi}_{L}\right)\left(\begin{array}{cccc}
-C^{\dagger} m_{R} & 0 & -\not D_{R}^{T} & -m_{L R}^{T} \\
0 & -C^{\dagger} m_{L} & -m_{R L}^{T} & -\not D_{L}^{T} \\
\not D_{R} & m_{R L} & C m_{R}^{\dagger} & 0 \\
m_{L R} & \not D_{L} & 0 & C m_{L}^{\dagger}
\end{array}\right)\left(\begin{array}{c}
\psi_{R} \\
\psi_{L} \\
\bar{\psi}_{R}^{T} \\
\bar{\psi}_{L}^{T}
\end{array}\right) .
$$

As it should be, the operator in between is antisymmetric, the fermion fields being Grassmann c-numbers. Functional integration on $\psi_{L, R}(x)$ and $\bar{\psi}_{L, R}(x)$ gives the Pffaffian of this operator, which equals the square root of its determinant. Having reduced the problem to a determinant, one can apply similarity transformations to remove $C$ to enforce explicit Lorentz invariance, as well as rearrangement of rows and of columns to obtain a suitable form. Specifically, we take

$$
\mathcal{L}(x)=\frac{1}{2}\left(\begin{array}{llll}
\bar{\psi}_{L} & \bar{\psi}_{R}^{c} & \bar{\psi}_{R} & \bar{\psi}_{L}^{c}
\end{array}\right)\left(\begin{array}{cccc}
m_{L R} & m_{L}^{\dagger} & \not D_{L} & 0 \\
m_{R} & m_{L R}^{T} & 0 & \not_{R}^{*} \\
\not D_{R} & 0 & m_{R L} & m_{R}^{\dagger} \\
0 & \not D_{L}^{*} & m_{L} & m_{R L}^{T}
\end{array}\right)\left(\begin{array}{c}
\psi_{R} \\
\psi_{L}^{c} \\
\psi_{L} \\
\psi_{R}^{c}
\end{array}\right):=\frac{1}{2} \bar{\Psi} \mathbf{D}_{2} \Psi
$$

It is important to remark that in eq. (2.30) $\not^{T}=\gamma_{\alpha}^{T} D_{\alpha}^{T}$, while in eq. (2.31) we use the notation $\not D^{*}:=\gamma_{\alpha} D_{\alpha}^{*}$ (no complex conjugation on the Dirac gammas). We will adopt the same notation in what follows. Also, $D_{\alpha}^{*}=\partial_{\alpha}+V_{\alpha}^{*}$ and $D_{\alpha}^{T}=-\partial_{\alpha}+V_{\alpha}^{T}=-D_{\alpha}^{*}$.

As already noted the partition function equals $\left(\operatorname{Det} \mathbf{D}_{2}\right)^{1 / 2}$, therefore

$$
\Gamma\left[m_{L R}, m_{R L}, m_{L}, m_{R}, V_{L}, V_{R}\right]=-\frac{1}{2} \operatorname{Tr} \log \mathbf{D}_{2} .
$$

The order of the fields in $\mathbf{D}_{2}$ has been chosen so that the problem of computing Det $\mathbf{D}_{2}$ is identical to that for pure Dirac fermions in eq. (2.8), with the replacements

$$
\begin{array}{ll}
\psi_{L, R} \rightarrow\left(\begin{array}{c}
\psi_{L, R} \\
\psi_{R, L}^{c}
\end{array}\right), & \bar{\psi}_{L, R} \rightarrow\left(\begin{array}{cc}
\bar{\psi}_{L, R} & \bar{\psi}_{R, L}^{c}
\end{array}\right), \\
m_{L R} \rightarrow\left(\begin{array}{cc}
m_{L R} & m_{L}^{\dagger} \\
m_{R} & m_{L R}^{T}
\end{array}\right), & m_{R L} \rightarrow\left(\begin{array}{cc}
m_{R L} & m_{R}^{\dagger} \\
m_{L} & m_{R L}^{T}
\end{array}\right), \\
D_{L \alpha} \rightarrow\left(\begin{array}{cc}
D_{L \alpha} & 0 \\
0 & D_{R \alpha}^{*}
\end{array}\right), & D_{R \alpha} \rightarrow\left(\begin{array}{cc}
D_{R \alpha} & 0 \\
0 & D_{L \alpha}^{*}
\end{array}\right) .
\end{array}
$$

In particular, the explicit results in [70-72] for the effective action of Dirac fermions can be immediately extended to Majorana fermions using the above identifications.

The chiral transformation of $\mathbf{D}_{2}$ is given by

$$
\mathbf{D}_{2} \rightarrow\left(\begin{array}{cccc}
\Omega_{L}^{\dagger} & & & \\
& \Omega_{R}^{T} & & \\
& & \Omega_{R}^{\dagger} & \\
& & & \Omega_{L}^{T}
\end{array}\right) \mathbf{D}_{2}\left(\begin{array}{cccc}
\Omega_{R} & & & \\
& & \Omega_{L}^{*} & \\
& & \Omega_{L} & \\
& & & \Omega_{R}^{*}
\end{array}\right)
$$


so the chiral invariant part of the effective action will involve the chiral covariant pieces in eq. (2.12), plus the new chiral covariant derivatives

$$
\hat{D}_{\alpha} m_{L}=D_{\alpha}^{L *} m_{L}-m_{L} D_{\alpha}^{L}, \quad \hat{D}_{\alpha} m_{R}=D_{\alpha}^{R *} m_{R}-m_{R} D_{\alpha}^{R} .
$$

\section{The (extended) Standard Model}

\subsection{Leptonic sector of the Standard Model}

The fermionic sector of the Standard Model of particle physics, extended to include either Dirac, Majorana or mixed neutrino masses can be accommodated in the scheme of the previous section. First we consider explicitly the general case where both Dirac and Majorana neutrino masses are present and eventually we will restrict ourselves to the simpler cases of pure Dirac or pure Majorana neutrino masses.

In order to apply eq. (2.31) to the leptonic sector of the extended Standard Model we take the identifications

$$
\bar{\psi}_{L, R}=\left(\bar{\nu}_{L, R} \bar{e}_{L, R}\right), \quad \psi_{L, R}=\left(\begin{array}{c}
\nu_{L, R} \\
e_{L, R}
\end{array}\right),
$$

where $e_{L, R}(x)$ is the field of the charged leptons. This is a Dirac spinor as well as a vector on generation or family space, containing the electron, muon and tau fields. Likewise, the vector $\nu_{L, R}(x)$ represents the fields of the three left-handed neutrinos, and a certain number $N_{s}$ of right-handed ones. The dimensions of $\nu_{L}, \nu_{R}, e_{L}$ and $e_{R}$ are $g, N_{s}, g$ and $g$, respectively, where $g=3$ is the number of generations.

Further, for the mass terms in eq. (2.31)

$$
m_{L R}=m_{R L}^{\dagger}=\left(\begin{array}{cc}
\frac{\phi}{v} M_{D} & 0 \\
0 & \frac{\phi}{v} M_{e}
\end{array}\right), \quad m_{L}=\left(\begin{array}{cc}
\frac{\phi^{2}}{v^{2}} M_{L} & 0 \\
0 & 0
\end{array}\right), \quad m_{R}=\left(\begin{array}{cc}
M_{R} & 0 \\
0 & 0
\end{array}\right) .
$$

$M_{D}$ and $M_{e}$ are constant complex matrices in generation space representing the Dirac mass matrices of neutrinos and charged leptons, respectively. Similarly $M_{L}$ and $M_{R}$ are the Majorana mass matrices of left and right handed neutrinos, respectively. The dimensions of $M_{D}, M_{e}, M_{L}$ and $M_{R}$ are $g \times N_{s}, g \times g, g \times g$ and $N_{s} \times N_{s}$, respectively.

We adopt the unitary gauge throughout. $\phi(x)$ is the neutral Higgs field in that gauge and $v$ its vacuum expectation value. The coupling of the mass terms to the Higgs field adopted here takes into account that $\bar{\nu}_{R} \nu_{L}$ and $\bar{e}_{R} e_{L}$ are $\mathrm{SU}(2)$ doublets, as the Higgs, while $\bar{\nu}_{L}^{c} \nu_{L}$ is a triplet and $\bar{\nu}_{R}^{c} \nu_{R}$ is a singlet [59].

Finally, the covariant derivatives in eq. (2.31), take the following form in the Standard Model:

$$
D_{\alpha}^{L}=\left(\begin{array}{cc}
D_{\alpha}^{\nu}+Z_{\alpha} & W_{\alpha}^{+} \\
W_{\alpha}^{-} & D_{\alpha}^{e}-Z_{\alpha}
\end{array}\right), \quad D_{\alpha}^{R}=\left(\begin{array}{cc}
D_{\alpha}^{\nu} & 0 \\
0 & D_{\alpha}^{e}
\end{array}\right) .
$$

$W_{\alpha}^{ \pm}$represent the fields of the charged bosons and $Z_{\alpha}$ the field of the $Z^{0}$. For convenience we have included the $\mathrm{SU}(2) \times \mathrm{U}(1)$ couplings in the gauge fields. The relation to the canonically normalized fields (denoted with tilde) is as follows [51, 78]

$$
W_{\alpha}^{ \pm}=\frac{1}{\sqrt{2}} g \tilde{W}_{\alpha}^{ \pm}, \quad Z_{\alpha}=\frac{1}{2} \frac{g}{\cos \theta_{W}} \tilde{Z}_{\alpha}=\frac{1}{2} g \tilde{W}_{\alpha}^{3}-\frac{1}{2} g^{\prime} \tilde{B}_{\alpha},
$$




\begin{tabular}{|ccc|}
\hline Quantity & $\begin{array}{c}\text { Complex } \\
\text { conjugation }\end{array}$ & CP \\
\hline$\phi$ & $\phi$ & $\phi$ \\
$W_{\alpha}^{ \pm}$ & $-W_{\alpha}^{\mp}$ & $-W_{\alpha}^{\mp}$ \\
$Z_{\alpha}$ & $-Z_{\alpha}$ & $-Z_{\alpha}$ \\
$\varphi_{\alpha}$ & $\varphi_{\alpha}$ & $\varphi_{\alpha}$ \\
$F_{\alpha \beta}^{e}$ & $-F_{\alpha \beta}^{e}$ & $-F_{\alpha \beta}^{e}$ \\
$\epsilon_{\mu \nu \alpha \beta}$ & $\epsilon_{\mu \nu \alpha \beta}$ & $-\epsilon_{\mu \nu \alpha \beta}$ \\
$U$ & $U^{*}$ & $U^{*}$ \\
\hline
\end{tabular}

Table 1. Transformation of various quantities (Euclidean version) under complex conjugation and $\mathrm{CP}$. The derivatives of the fields follow the same rules as the fields themselves.

where $\tilde{B}_{\alpha}$ is the gauge field of the weak hypercharge $\mathrm{U}(1)$ group and $\theta_{W}$ the weak angle. On the other hand, $D_{\nu}$ and $D_{e}$ are covariant derivatives, corresponding to the remaining gauge freedom within the unitary gauge. Specifically,

$$
\begin{array}{ll}
D_{\alpha}^{\nu}=\partial_{\alpha}, & A_{\alpha}^{\nu}=0, \\
D_{\alpha}^{e}=\partial_{\alpha}+A_{\alpha}^{e}, & A_{\alpha}^{e}=-g^{\prime} \tilde{B}_{\alpha}=-e \tilde{A}_{\alpha}+2 \sin ^{2} \theta_{W} Z_{\alpha},
\end{array}
$$

where $\tilde{A}_{\alpha}$ is the photon field and $-e$ the electron electric charge. In the following we will work with $Z_{\alpha}$ and $D_{\alpha}^{e}$ as basic variables, but it should be remembered that $Z_{\alpha}$ also appears in $D_{\alpha}^{e}$ when the final results are expressed in terms of the physical fields $\tilde{Z}_{\alpha}$ and $\tilde{A}_{\alpha}$.

From the general formula in eq. (2.12) the following field strengths can be constructed:

$$
\begin{aligned}
W_{\alpha \beta}^{+} & :=D_{\alpha}^{\nu} W_{\beta}^{+}-W_{\beta}^{+} D_{\alpha}^{e}=\partial_{\alpha} W_{\beta}^{+}-A_{\alpha}^{e} W_{\beta}^{+}, \\
W_{\alpha \beta}^{-} & :=D_{\alpha}^{e} W_{\beta}^{-}-W_{\beta}^{-} D_{\alpha}^{\nu}=\partial_{\alpha} W_{\beta}^{-}+A_{\alpha}^{e} W_{\beta}^{-}, \\
F_{\alpha \beta}^{e} & :=\left[D_{\alpha}^{e}, D_{\beta}^{e}\right]=\partial_{\alpha} A_{\beta}^{e}-\partial_{\beta} A_{\alpha}^{e}, \\
Z_{\alpha \beta} & :=D_{\alpha}^{\nu} Z_{\beta}-Z_{\beta} D_{\alpha}^{\nu}=\partial_{\alpha} Z_{\beta} .
\end{aligned}
$$

Let us emphasize that the tensors $W_{\alpha \beta}^{ \pm}$and $Z_{\alpha \beta}$ just defined are not antisymmetric.

The properties of these fields under complex conjugation and CP follow from eqs. (2.3) and (2.5). They are summarized in table 1.

\subsection{Neutrinos with mixed Dirac-Majorana mass terms}

The main topic of the paper is Majorana and Dirac neutrinos. Nevertheless, for future reference, in the rest of this section we briefly review the case of general neutrino mass matrices. For simplicity we set $\phi(x)=v$ in this discussion and assume that there are no accidental mass degeneracies and no fermion is massless.

Certainly, one can rotate the lepton fields by means of constant unitary matrices so that the eqs. (3.2) and (3.3) are unchanged except that $M_{e}$ is replaced by a diagonal and positive matrix $m_{e}$. Once this choice is taken, the neutrino mass matrices can be 
diagonalized by means of a unitary transformation as follows

$$
\left(\begin{array}{ll}
M_{L}^{*} & M_{D} \\
M_{D}^{T} & M_{R}
\end{array}\right)=\mathcal{U}\left(\begin{array}{cc}
m_{1} & 0 \\
0 & m_{2}
\end{array}\right) \mathcal{U}^{T}, \quad \mathcal{U}=\left(\begin{array}{cc}
A & B \\
D & C
\end{array}\right), \quad \mathcal{U}^{-1}=\mathcal{U}^{\dagger}
$$

so that the matrices $m_{1,2}$ are diagonal and positive. $A$ and $C$ are square submatrices but need not be unitary.

If these rotations are applied to the fermion fields the structure in eq. (2.31) is not preserved, since right and left handed fields are mixed. However the form in eq. (2.8) still holds, with

$$
\begin{aligned}
m_{L R}= & m_{R L}^{\dagger}=\left(\begin{array}{cccc}
0 & 0 & m_{1} & 0 \\
0 & m_{e} & 0 & 0 \\
m_{2} & 0 & 0 & 0 \\
0 & 0 & 0 & m_{e}
\end{array}\right), \\
D_{\alpha}^{L}= & \left(\begin{array}{cccc}
D_{\alpha}^{\nu}+A^{\dagger} A Z_{\alpha} & A^{\dagger} W_{\alpha}^{+} & A^{\dagger} B Z_{\alpha} & 0 \\
A W_{\alpha}^{-} & D_{\alpha}^{e}-Z_{\alpha} & B W_{\alpha}^{-} & 0 \\
B^{\dagger} A Z_{\alpha} & B^{\dagger} W_{\alpha}^{+} & D_{\alpha}^{\nu}+B^{\dagger} B Z_{\alpha} & 0 \\
0 & 0 & 0 & D_{\alpha}^{e, *}
\end{array}\right), \\
D_{\alpha}^{R}= & \left(\begin{array}{cccc}
D_{\alpha}^{\nu}-B^{T} B^{*} Z_{\alpha} & 0 & -B^{T} A^{*} Z_{\alpha} & -B^{T} W_{\alpha}^{-} \\
0 & D_{\alpha}^{e} & 0 & 0 \\
-A^{T} B^{*} Z_{\alpha} & 0 & D_{\alpha}^{\nu}-A^{T} A^{*} Z_{\alpha} & -A^{T} W_{\alpha}^{-} \\
-B^{*} W_{\alpha}^{+} & 0 & -A^{*} W_{\alpha}^{+} & D_{\alpha}^{e, *}+Z_{\alpha}
\end{array}\right) .
\end{aligned}
$$

Here the fermion fields of eq. (2.8) correspond to $\psi_{R}=\left(\nu_{R}, e_{R}, \nu_{L}^{c}, e_{L}^{c}\right), \psi_{L}=$ $\left(\nu_{L}, e_{L}, \nu_{R}^{c}, e_{R}^{c}\right), \bar{\psi}_{R}=\left(\bar{\nu}_{R}, \bar{e}_{R}, \bar{\nu}_{L}^{c}, \bar{e}_{L}^{c}\right)$, and $\bar{\psi}_{L}=\left(\bar{\nu}_{L}, \bar{e}_{L}, \bar{\nu}_{R}^{c}, \bar{e}_{R}^{c}\right)$. (For simplicity, we denote rotated and unrotated lepton fields with the same symbols, as we are not using them in the rest of the paper.)

It is noteworthy that the Dirac operator in eq. (3.8) is independent of the submatrices $C$ and $D$ in $\mathcal{U}$. In addition, the determinant of that Dirac operator, and consequently its effective action, is unchanged if the matrices $A$ and $B$ are subject to the following transformations:

$$
(A, B) \rightarrow\left(V A V_{A}, V B V_{B}\right), \quad V=\operatorname{diag}\left(e^{\theta_{1}}, \ldots, e^{\theta_{g}}\right), \quad V_{A}, V_{B}=\operatorname{diag}( \pm, \ldots, \pm) .
$$

(Here $g$ denotes the number of generations, and the diagonal matrices $V_{A}$, and $V_{B}$ have dimension $g$ and $N_{s}$, respectively.)

In the limit of large $M_{R}$ keeping $M_{D}$ and $M_{L}$ finite, $B$ vanishes and $A$ becomes unitary. In this case the neutrinos with mass $m_{2}$ (denoted $\nu_{R}$ above) completely decouple and only left-handed neutrinos remain. This is equivalent to assuming neutrinos with pure Majorana masses (i.e., $M_{D}=0$ ) from the beginning. If in addition to large $M_{R}, M_{L}=0$ is also assumed, $m_{1}$ is small, which is the well-known seesaw mechanism to account for the small masses of the neutrinos [57-60]. The coupling to the Higgs field assumed in eq. (3.2) is also consistent with this scenario. The coupling between charged and neutral leptons takes the form $\bar{e}_{L} A W^{-} \nu_{L}$, therefore, in this limit, $A$ is identified with the PMNS matrix [33-35] usually denoted by $U$ in the literature [36]. 

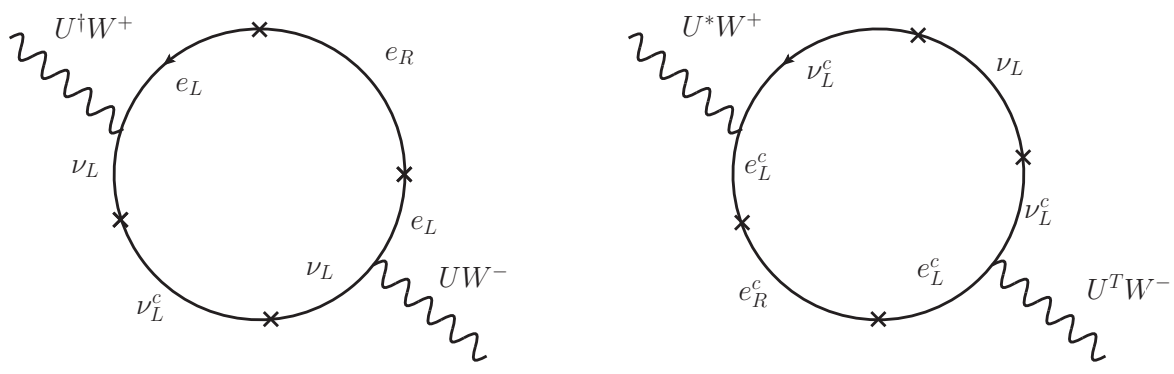

Figure 1. Mechanisms present in $\Gamma_{2}$. Gauge bosons are inwards.

\section{CP odd component of the effective action}

As said, the effective action $\Gamma\left[m_{L R}, m_{R L}, m_{L}, m_{R}, V_{L}, V_{R}\right]$ in eq. (2.32) is invariant under a full CP transformation applied to all external fields. In the Standard Model the physical $\mathrm{CP}$ transformation refers to the gauge fields and Higgs (and to fermions but these are integrated out in the effective action) while the fermion mass matrices are unchanged. So, to identify the even and odd components of $\Gamma$ under $\mathrm{CP}$, one can look for the symmetric and antisymmetric components when the gauge fields and Higgs are CP-transformed or, more conveniently, when the mass matrices are CP-transformed. From eqs. (2.5) and (2.29), the latter amounts to

$$
M_{L} \rightarrow M_{L}^{*}, \quad M_{R} \rightarrow M_{R}^{*}, \quad M_{D} \rightarrow M_{D}^{*}, \quad M_{e} \rightarrow M_{e}^{*}
$$

After diagonalization, for Majorana or Dirac neutrinos, the previous transformation amounts to

$$
U \rightarrow U^{*} \quad \text { (CP transformation) }
$$

For pure Majorana neutrinos $(A=U$ and $B=0$ in eq. (3.8)) or pure Dirac neutrinos ( $A=0$ and $B=U$ ), it follows that the presence of $U$ is always tied to a charged current vertex. Therefore, the CP violating component of $\Gamma$ must contain $W^{ \pm}$fields, and in equal number of $W^{+}$and $W^{-}$, in order to fulfill electric charge conservation, since there are no other charged external fields. Thus we will consider an expansion of the effective action in powers of the $W^{ \pm}$fields:

$$
\Gamma=\sum_{n=0}^{\infty} \Gamma_{2 n},
$$

where $\Gamma_{2 n}$ contains $n W^{+} W^{-}$pairs.

From the previous discussion it follows that $\Gamma_{0}$ cannot have a $\mathrm{CP}$ violating component. The same is true of $\Gamma_{2}$. Indeed, within the formulation on the Dirac operator of the previous section, the two possible types of Feynman graphs for $\Gamma_{2}$ are those displayed in figure 1 . It is sufficient to consider one of them since they are related by conjugation and give the same result. In generation space, a typical graph has a structure

$$
G(U)=\operatorname{tr}\left(U^{\dagger} f_{1}\left(m_{e}\right) U f_{2}\left(m_{\nu}\right)\right)
$$


from the vertices $U^{\dagger} W^{+}$and $U W^{-}$, and $f_{1}\left(m_{e}\right)$ and $f_{2}\left(m_{\nu}\right)$ are diagonal matrices from the propagators. Under CP,

$$
\begin{aligned}
G(U) \rightarrow G\left(U^{*}\right) & =\operatorname{tr}\left(U^{T} f_{1}\left(m_{e}\right) U^{*} f_{2}\left(m_{\nu}\right)\right)=\operatorname{tr}\left(\left(U^{T} f_{1}\left(m_{e}\right) U^{*} f_{2}\left(m_{\nu}\right)\right)^{T}\right) \\
& =\operatorname{tr}\left(f_{2}\left(m_{\nu}\right) U^{\dagger} f_{1}\left(m_{e}\right) U\right)=G(U)
\end{aligned}
$$

This result was to be expected: $\Gamma_{2}$ is structurally identical for Majorana and Dirac neutrinos, or even for leptons and quarks. As is well-known, the insertion of just one $W^{+}$ and one $W^{-}$in the quark loop does not allow the quarks to visit the three generations, which is the minimum required to have $\mathrm{CP}$ violation with Dirac particles [32]. Beyond $\Gamma_{2}$ it is no longer true that the Feynman graphs involving Majorana and Dirac neutrinos have necessarily the same structure. In the Majorana case, fermionic number violating terms appear in $\Gamma_{4}$ that allow to break CP even for two generations.

Of course, to reach the conclusion that $\Gamma_{2}$ is $\mathrm{CP}$ even it is crucial that we are considering only the one-loop effective action. It is perfectly possible to write $\mathrm{CP}$ violating operators of the type $W^{+} W^{-}$. For instance

$$
Z_{\alpha}\left(W_{\alpha}^{+} W_{\beta \beta}^{-}+W_{\beta \beta}^{+} W_{\alpha}^{-}\right), \quad \epsilon_{\mu \nu \alpha \beta} W_{\mu \nu}^{+} W_{\alpha \beta}^{-} .
$$

The first one is parity even, the second one is parity odd. Our previous argument implies that such operators require Feynman graphs with internal gauge boson lines, and this amounts to going beyond one-loop.

We will further use the notation $\Gamma_{2 n+d}$ to indicate the component of the effective action composed of operators with $n W^{+} W^{-}$pairs and a total of $2 n+d$ Lorentz indices carried by the fields. So the two operators in eq. (4.6) are of the type $2+2$. Within a covariant derivative expansion, $2 n+d$ is the order of the operator, that is, the number of derivatives it carries (in this counting each gauge field or derivative counts as order 1, the Higgs field is of order 0). Equivalently, $2 n+d$ is the dimension of the operator (counting the operator $\phi / v$ as dimensionless). In an even-dimensional spacetime $d$ is always even.

We have just argued that $\Gamma_{0+d}$ and $\Gamma_{2+d}$ are CP even for any value of $d$. It is easy to see that the components $\Gamma_{2 n+0}$ are also CP even. Indeed, no CP odd operator can be written using only $W^{ \pm}$with no other gauge fields nor derivatives [51]. Since operators of the type $2 n+d>4$ are UV convergent in four dimensions, this implies that all UV divergent terms of the effective action are $\mathrm{CP}$ even. This includes the gauged WZW term which has dimension four. The first contribution to $\mathrm{CP}$ violation comes from the dimension 6 operators in $\Gamma_{4+2}$ (as said $\Gamma_{6+0}$ is CP even). These are the operators to be considered in this work, specifically for Majorana neutrinos. $\Gamma_{4+2}$ for quarks have been computed in $[51,52]$. Some operators of the type $4+4$ have been calculated for the quark sector in [53] and those of the type $6+2$ in [54].

The effective action can be expanded in the form

$$
\Gamma=\int d^{4} x \sum_{k}\left(\frac{v}{\phi(x)}\right)^{d_{k}-4} g_{k} \mathcal{O}_{k}(x),
$$



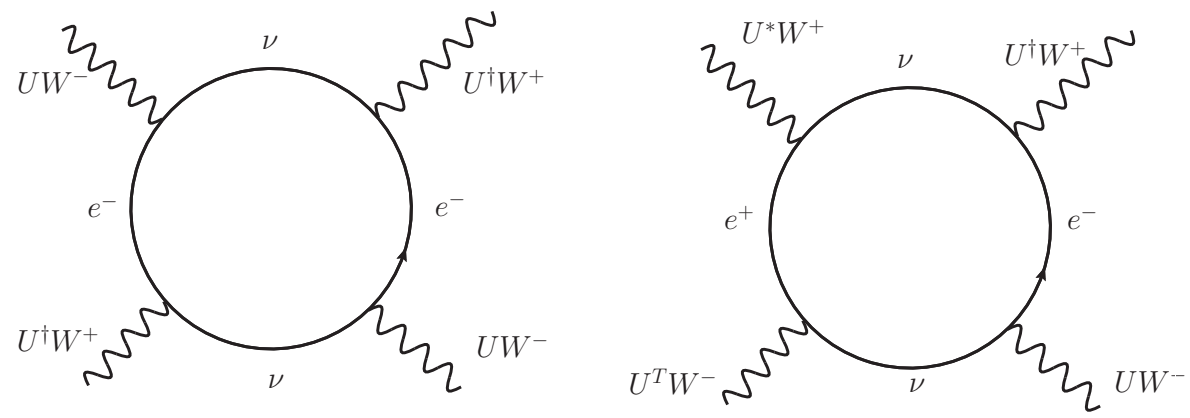

Figure 2. Mechanisms involved in $\Gamma_{4 a}$ (left panel) and $\Gamma_{4 b}$ (right panel). Gauge bosons are inwards.

where the $\mathcal{O}_{k}$ represent local operators of dimension $d_{k}=2 n+d$, constructed with gauge fields and their derivatives, as well as derivatives of the Higgs field. The $g_{k}$ are the corresponding couplings and they depend on the lepton mass matrices. The couplings come as integrals over the momentum of the fermion running in the loop. If underivated $\phi(x)$ are not included in the operators, they should go in the couplings. However, for Dirac neutrinos, the dependence on underivated $\phi$ follows from dimensional counting since the Higgs couples as the fermion masses, and this produces the explicit dependence shown in (4.7). For Majorana neutrinos, this is no longer true and the $g_{k}$, as defined in (4.7), still retain some dependence on $\phi(x)$ from the neutrino masses.

An operator $\mathcal{O}_{k}$ is even under charge conjugation if and only it is hermitian, therefore the $\mathrm{CP}$ odd operators are antihermitian in the parity even sector and hermitian in the parity odd one (table 1). Recalling that $\Gamma^{+}$is real and $\Gamma^{-}$is imaginary, it follows that the couplings of CP violating operators are purely imaginary. The same conclusion follows from noting that in Euclidean space no factor $i$ (imaginary unit) is generated through the Feynman rules, momentum integration or tracing of Dirac gammas, hence $g_{k}$ will be imaginary if and only if it is antisymmetric under $U \rightarrow U^{*}$.

As said, the first term with a $\mathrm{CP}$ odd component is $\Gamma_{4}$. The two mechanisms involved there are displayed in figure 2 and they correspond to two types of momentum integrals, $I_{a}$ and $I_{b}$,

$$
\begin{aligned}
& I_{a, n_{e}, n_{\nu}, n_{e}^{\prime}, n_{\nu}^{\prime}}^{k}=\operatorname{Im} \int \frac{d^{4} p}{(2 \pi)^{4}}\left(p^{2}\right)^{k / 2} \operatorname{tr}\left(N_{e}^{n_{e}} N_{\nu}^{n_{\nu}} N_{e}^{n_{e}^{\prime}} N_{\nu}^{n_{\nu}^{\prime}}\right) \\
& I_{b, n_{e}, n_{\nu}, n_{e}^{\prime}, n_{\nu}^{\prime}}^{k}=\operatorname{Im} \int \frac{d^{4} p}{(2 \pi)^{4}}\left(p^{2}\right)^{k / 2} \operatorname{tr}\left(N_{e}^{n_{e}} m_{\nu} N_{\nu}^{n_{\nu}} N_{e}^{* n_{e}^{\prime}} m_{\nu} N_{\nu}^{n_{\nu}^{\prime}}\right)
\end{aligned}
$$

where the exponents $n_{e}, n_{\nu}, n_{e}^{\prime}, n_{\nu}^{\prime}$ are natural numbers,

$$
N_{e}=U^{\dagger} \frac{1}{p^{2}+m_{e}^{2}} U, \quad N_{\nu}=\frac{1}{p^{2}+m_{\nu}^{2}},
$$

and $m_{e}$ and $m_{\nu}$ denote the positive and diagonal mass matrices of charged and neutral leptons, respectively. The $\mathrm{CP}$ odd sector only makes use of the imaginary parts of the 


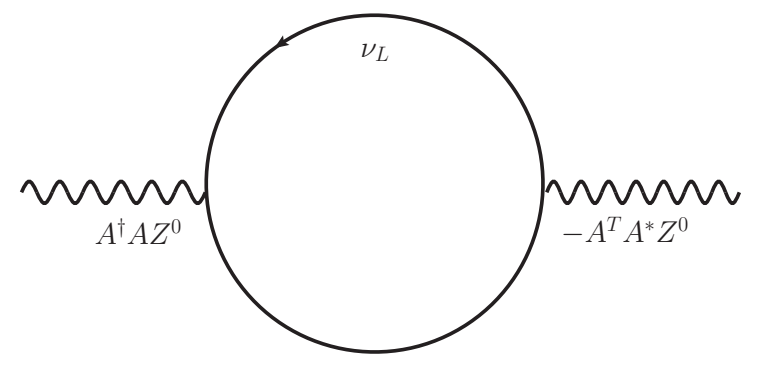

Figure 3. Graph contributing to $\mathrm{CP}$ violation with only neutral particles, provided the neutrinos have mixed Dirac-Majorana masses.

integrals. For these integrals, the following symmetries are easily established

$$
\begin{aligned}
I_{a, n_{e}, n_{\nu}, n_{e}^{\prime}, n_{\nu}^{\prime}}^{k} & =-I_{a, n_{e}^{\prime}, n_{\nu}, n_{e}, n_{\nu}^{\prime}}^{k}=-I_{a, n_{e}, n_{\nu}^{\prime}, n_{e}^{\prime}, n_{\nu}}^{k}, \\
I_{b, n_{e}, n_{\nu}, n_{e}^{\prime}, n_{\nu}^{\prime}}^{k} & =+I_{b, n_{e}^{\prime}, n_{\nu}, n_{e}, n_{\nu}^{\prime}}^{k}=-I_{b, n_{e}, n_{\nu}^{\prime}, n_{e}^{\prime}, n_{\nu} .} .
\end{aligned}
$$

Using the relations (11.6) and (11.7) of [51], the basic momentum integrals required to obtain $I_{a}$ and $I_{b}$ can be reduced to contour integrals which are easily computed by residues.

Before closing this section, we remark that the necessity of charged gauge bosons in the fermion loop to produce $\mathrm{CP}$ violation follows from the fact that the complex mass matrices are summarized into $m_{e}, m_{\nu}$ and $U$. The first two are real and the latter appears though the operator $\bar{e} U W^{-} \nu$ and its hermitian conjugate. This is true for pure Dirac or pure Majorana neutrinos, but it no longer holds in the general case of mixed Dirac and Majorana masses. Indeed, using the couplings in eq. (3.8) one can construct CP odd graphs involving no charged boson. One such graph is displayed in figure 3. The driving operator there is of the type

$$
i \epsilon_{\mu \nu \alpha \beta} Z_{\mu \nu} Z_{\alpha \beta} \operatorname{Im} \operatorname{tr}\left(A^{\dagger} A f_{1}\left(m_{\nu}\right) A^{T} A^{*} f_{2}\left(m_{\nu}\right)\right)
$$

where $f_{1}\left(m_{\nu}\right)$ and $f_{2}\left(m_{\nu}\right)$ are real and diagonal mass matrices. Here $m_{\nu}$ refers to $m_{1}$ of eq. (3.7). Similar terms appear with $m_{2}$ and $B$ at this fourth order. These operators can give a CP violating contribution for $g \geq 2$ and $N_{s} \geq 1$. Of course, in the limiting Dirac or Majorana cases $A$ and $B$ are unitary or zero and these couplings vanish.

The operator in (4.11) is $\mathrm{P}$ odd and of dimension 4, so the coupling would be logarithmically UV divergent, however, the dimension 4 refers only to the derivatives. In fact, the Higgs field is present in $m_{\nu}$. After expanding in powers of the fluctuation of the Higgs field, $\phi-v$, the order zero is a total derivative and the higher orders in $\phi-v$ are non renormalizable and UV convergent.

\section{Operator $\mathrm{K}$ for Majorana neutrinos}

The case of Dirac fermions has been addressed in [51] for quarks. The results obtained there translate almost immediately to the case of Dirac neutrinos, therefore we give more details of the derivation of the Majorana case, which is also more involved. 
We consider pure Majorana left-handed neutrinos, that is, $M_{D}=0$. Hypothetical right-handed neutrinos can be disregarded since they decouple from the other fields. In this case, the Lagrangian in the form given in eq. (2.31), with the identifications in eqs. (3.1)-(3.3), depends on the following Dirac operator

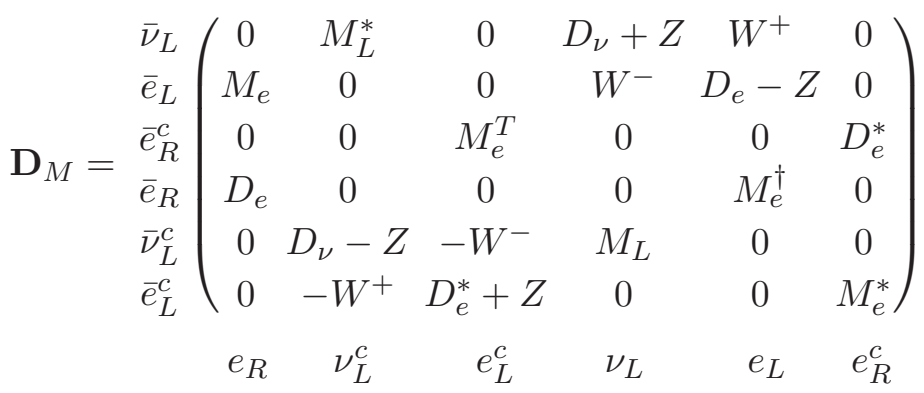

The fields associated to each row and column of the matrix are also displayed. The rows and columns associated to the fields $\nu_{R}, \bar{\nu}_{R}, \nu_{R}^{c}$ and $\bar{\nu}_{R}^{c}$ have been dropped.

$M_{L}$ and $M_{e}$ are complex $3 \times 3$ matrices which we assume to be regular (any massless case should be obtained as a limit) and $M_{L}$ is symmetric. The diagonalization of these mass matrices is addressed below.

In eq. (5.1) the contraction of fourvectors with the Dirac gamma matrices is understood and not explicitly displayed. In addition, to avoid clumsiness we have included the Higgs field factors in the mass matrices, so $M_{L}$ really stands for $(\phi / v)^{2} M_{L}$ and $M_{e}$ stands for $(\phi / v) M_{e}$. This means that $M_{L, e}$ are not constant, rather

$$
\left[\partial_{\mu}, M_{L}\right]=2 \varphi_{\mu}(x) M_{L}, \quad\left[\partial_{\mu}, M_{e}\right]=\varphi_{\mu}(x) M_{e},
$$

where we have introduced the auxiliary Higgs field

$$
\varphi_{\mu}(x):=\frac{\partial_{\mu} \phi(x)}{\phi(x)}
$$

Before proceeding let us make a small digression. It is clear that $\mathbf{D}_{M}$ above contains some redundant information, since the duplication of the charged lepton field (first $e_{L, R}$ and $\bar{e}_{L, R}$ and then $e_{L, R}^{c}$ and $\bar{e}_{L, R}^{c}$ ), being of Dirac type, is not strictly necessary. Indeed, the Lagrangian can be written in matrix form as

$$
\begin{aligned}
\mathcal{L}(x) & =\left(\begin{array}{llll}
\bar{\nu}_{L} & \bar{\nu}_{L}^{c} & \bar{e}_{L} & \bar{e}_{R}
\end{array}\right)\left(\begin{array}{cccc}
\frac{1}{2}\left(D_{\nu}+Z\right) & \frac{1}{2} M_{L}^{*} & W^{+} & 0 \\
\frac{1}{2} M_{L} & \frac{1}{2}\left(D_{\nu}-Z\right) & 0 & 0 \\
W^{-} & 0 & D_{e}-Z & M_{e} \\
0 & 0 & M_{e}^{\dagger} & D_{e}
\end{array}\right)\left(\begin{array}{c}
\nu_{L} \\
\nu_{L}^{c} \\
e_{L} \\
e_{R}
\end{array}\right) \\
& =\left(\begin{array}{llll}
\bar{\nu}_{L} & \bar{\nu}_{L}^{c} & \bar{e}_{L} & \bar{e}_{R}
\end{array}\right)\left(\begin{array}{cccc}
\frac{1}{2}\left(D_{\nu}+Z\right) & \frac{1}{2} m_{\nu} & U^{\dagger} W^{+} & 0 \\
\frac{1}{2} m_{\nu} & \frac{1}{2}\left(D_{\nu}-Z\right) & 0 & 0 \\
U W^{-} & 0 & D_{e}-Z & m_{e} \\
0 & 0 & m_{e}^{\dagger} & D_{e}
\end{array}\right)\left(\begin{array}{c}
\nu_{L} \\
\nu_{L}^{c} \\
e_{L} \\
e_{R}
\end{array}\right) .
\end{aligned}
$$

In the second form $m_{e}$ and $m_{\nu}$ are diagonal and positive, namely, by taking $M_{L}=U^{*} m_{\nu} U^{\dagger}$ in the basis in which $M_{e}$ is diagonal. This form of the Lagrangian is of course correct, 


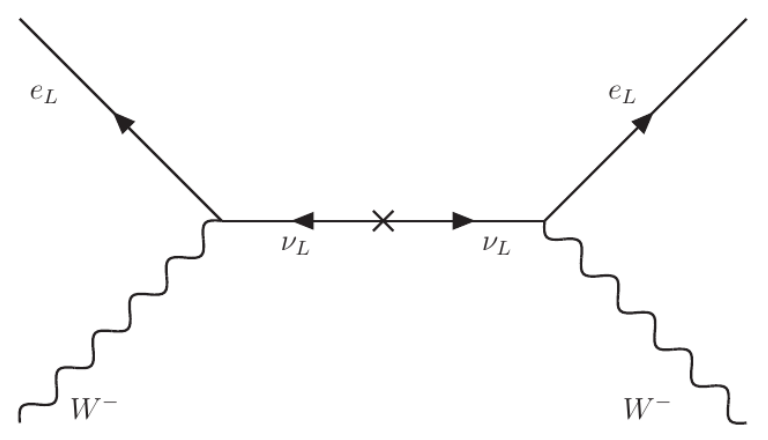

Figure 4. Feynman graph corresponding to eq. (5.5).

however, it involves both real and complex Grassman fields. As a consequence the effective action is not obtained as the determinant of the associated Dirac operator. For instance, such determinant does not contain contributions with $U$ and $U^{T}$ simultaneously, which are actually present in the effective action (namely, through the integrals $I_{b}$ in (4.8)). These terms are correctly generated by the Lagrangian in eq. (5.4) taking into account that the Wick contraction of $\nu_{L}$ and $\nu_{L}^{c}$ is not vanishing (see figure 4):

$$
(\bar{e}_{L} U W^{-} \underbrace{}_{L})\left(\bar{\nu}_{L} m_{\nu} \nu_{L}^{c}\right)\left(\bar{e}_{L} U W^{-} \nu_{L}\right) \sim \bar{e}_{L} U W^{-} f\left(m_{\nu}\right) U^{T} W^{-} e_{L}^{c} .
$$

On the contrary, within the formalism based on (5.1) only contractions of the type $\psi \bar{\psi}$ (rather than $\psi \psi$ or $\bar{\psi} \bar{\psi}$ ) are required. We find it preferable to duplicate all fields thereby reducing the calculation of the effective action to that of a determinant.

Coming back to (5.1), since $\mathbf{D}_{M}$ is of the general form in eq. (2.8) we can proceed to construct the auxiliary operators $K_{L, R}$ of eq. (2.19). As already noted, the two operators contain the same information for UV convergent contributions to the effective action. In what follows we use $K_{L}$. The inverse of $m_{R L}$ is readily obtained:

$$
m_{R L}^{-1}=\left(\begin{array}{ccc}
0 & M_{L}^{-1} & 0 \\
M_{e}^{\dagger-1} & 0 & 0 \\
0 & 0 & M_{e}^{*-1}
\end{array}\right)
$$

A straightforward calculation then gives

$$
K_{L}=\left(\begin{array}{ccc}
M_{L}^{\dagger} M_{L}-\left(D_{\nu}+Z\right)\left(D_{\nu}-Z+2 \varphi\right) & -W^{+}\left(D_{e}+\varphi\right) & \left(D_{\nu}+Z\right) W^{-} M_{L}^{-1} M_{e}^{*} \\
-W^{-}\left(D_{\nu}-Z+2 \varphi\right) & M_{e} M_{e}^{\dagger}-\left(D_{e}-Z\right)\left(D_{e}+\varphi\right) & W^{-} W^{-} M_{L}^{-1} M_{e}^{*} \\
D_{e}^{*} W^{+} M_{e}^{*-1} M_{L} & 0 & M_{e}^{T} M_{e}^{*}-D_{e}^{*}\left(D_{e}^{*}+Z+\varphi\right)
\end{array}\right)
$$

In terms of $K_{L}$, the effective action is given by

$$
\Gamma=-\frac{1}{2} \operatorname{Tr}\left(P_{R} \log K_{L}\right)
$$


where the factor $1 / 2$ is that in eq. (2.32), and it takes into account that all fields have been duplicated in order to achieve a Lagrangian formally of Dirac type (that is, with effective action directly related to the determinant of the differential operator).

We remark that the matrix elements displayed in (5.7) are themselves $4 \times 4$ matrices in Dirac space and $g \times g$ matrices in generation space, for $g$ generations. Also, the fields naturally involved there are $\left(\nu_{L}^{c}, e_{R}, e_{L}^{c}\right)$ and $\left(\bar{\nu}_{L}, \bar{e}_{L}, \bar{e}_{R}^{c}\right)$. More precisely, from eq. (2.26) (adapted to include the duplication required by Majorana terms) it follows that the propagator of these fields is correctly reproduced by the effective Lagrangian

$$
\mathcal{L}^{\mathrm{eff}}(x)=\left(\begin{array}{lll}
\bar{\nu}_{L} & \bar{e}_{L} & \bar{e}_{R}^{c}
\end{array}\right) K_{L}\left(\begin{array}{c}
M_{L}^{-1} \nu_{L}^{c} \\
M_{e}^{\dagger-1} e_{R} \\
M_{e}^{*-1} e_{L}^{c}
\end{array}\right) .
$$

Since for the effective action all we needed is $\operatorname{Det}\left(P_{R} K_{L}\right)$, there remains the freedom to apply similarity transformations to $K_{L}$ to obtain a more convenient form. To this end, we multiply $K_{L}$ on the left by the matrix $\phi^{-1} \operatorname{diag}\left(M_{L}, M_{L}, M_{e}^{*}\right)$, and its inverse on the right. This produces the equivalent operator

$$
K_{1}=\left(\begin{array}{ccc}
M_{L} M_{L}^{\dagger}-D_{\nu+} D_{\nu-} & -W^{+} D_{e} & D_{\nu+} W^{-} \\
-W^{-} D_{\nu-} & M_{L} M_{e} M_{e}^{\dagger} M_{L}^{-1}-D_{e-} D_{e} & W^{-} W^{-} \\
D_{e}^{*} W^{+} & 0 & M_{e}^{*} M_{e}^{T}-D_{e}^{*} D_{e+}^{*}
\end{array}\right)
$$

where we have defined the following shorthands

$$
D_{\nu \pm} \equiv D_{\nu} \pm(Z-\varphi), \quad D_{e \pm} \equiv D_{e} \pm(Z+\varphi), \quad D_{e \pm}^{*} \equiv D_{e}^{*} \pm(Z+\varphi) .
$$

Below we will also make use of the notation

$$
X^{(n)} \equiv \phi^{-n} X \phi^{n}, \quad D_{\mu}^{(n)}=\phi^{-n} D_{\mu} \phi^{n}=D_{\mu}+n \varphi_{\mu},
$$

where $X$ is a generic quantity and in particular $D_{\mu}=\partial_{\mu}+V_{\mu}$ is any derivative operator.

It is noteworthy that in the neutrino sector only the combination $Z-\varphi$ appears, and only $Z+\varphi$ in the charged sector, and moreover, the same property holds true for the Dirac case [51]. We have no a priori explanation for this regularity. One should note however that this property needs not translate immediately to the effective action since new $\varphi_{\mu}$ can be generated through the $\phi$ dependence contained in $M_{L}$ and $M_{e}$.

For comparison, we quote here the similar operator $K_{L}$ for Dirac neutrinos [51]: ${ }^{6}$

$$
K_{\text {Dirac }}=\left(\begin{array}{cc}
M_{D} M_{D}^{\dagger}-D_{\nu+} D_{\nu} & -W^{+} D_{e} \\
-W^{-} D_{\nu} & M_{e} M_{e}^{\dagger}-D_{e-} D_{e}
\end{array}\right),
$$

where $D_{\nu}=\partial$ and the Dirac neutrino mass matrix $M_{D}$ includes a factor $\phi / v$. When this is regarded as an effective Lagrangian, the fermion fields involved in this matrix are $\left(\bar{\nu}_{L}, \bar{e}_{L}\right)$ and $\left(\begin{array}{c}M_{D}^{-1} \nu_{R} \\ M_{e}^{-1} e_{R}\end{array}\right)$

\footnotetext{
${ }^{6}$ We have applied a similarity transformation $K \rightarrow \phi K \phi^{-1}$ to the matrix of eq. (7.1) of [51], in order to enforce the dependence $Z \pm \varphi$ for the neutrino (up) and charged (down) sectors.
} 
To proceed with the Majorana case we bring the fermion masses to a diagonal form:

$$
M_{L}=A m_{\nu} A^{T}, \quad M_{e}=B m_{e} C, \quad U:=B^{\dagger} A^{*} .
$$

Here $A, B, C$ are suitable constant unitary matrices such that $m_{\nu}$ and $m_{e}$ are positive diagonal matrices (which include factors $(\phi / v)^{2}$ and $\phi / v$, respectively). Multiplying $K_{1}$ in (5.10) by $\operatorname{diag}\left(A^{\dagger}, A^{\dagger}, A^{\dagger}\right)$ on the left, and its inverse on the right, produces the equivalent matrix (also denoted by $K_{1}$ )

$$
K_{1}=\left(\begin{array}{ccc}
m_{\nu}^{2}-D_{\nu+} D_{\nu-} & -W^{+} D_{e} & D_{\nu+} W^{-} \\
-W^{-} D_{\nu-} & m_{\nu} U^{\dagger} m_{e}^{2} U m_{\nu}^{-1}-D_{e-} D_{e} & W^{-} W^{-} \\
D_{e}^{*} W^{+} & 0 & U^{T} m_{e}^{2} U^{*}-D_{e}^{*} D_{e+}^{*}
\end{array}\right) .
$$

The determinant of $K_{1}$ is unchanged by transformations of $U$ of the type

$$
U \rightarrow \operatorname{diag}\left(e^{\theta_{1}}, \cdots, e^{\theta_{g}}\right) U \operatorname{diag}( \pm, \ldots, \pm)
$$

and these are the unique allowed transformations if masses are not degenerated.

If the expression in (5.15) is used directly in the calculation of the effective action, inverse powers of $m_{\nu}$ appear in intermediate steps, although eventually they can be removed in every single case. In order to obtain directly the expressions without $m_{\nu}^{-1}$, we proceed as follows. First we define the following propagators

$$
\begin{aligned}
G_{\nu} & :=\left(m_{\nu}^{2}-D_{\nu+} D_{\nu-}\right)^{-1}, \\
G_{e} & :=U^{\dagger}\left(m_{e}^{2}-D_{e-} D_{e}\right)^{-1} U, \\
G_{e}^{*} & :=U^{T}\left(m_{e}^{2}-D_{e}^{*} D_{e+}^{*}\right)^{-1} U^{*} .
\end{aligned}
$$

In terms of these, the matrix $K_{1}$ can be expressed as

$$
K_{1}=\left(\begin{array}{ccc}
G_{\nu}^{-1} & -W^{+} D_{e} & D_{\nu+} W^{-} \\
-W^{-} D_{\nu-} & m_{\nu} G_{e}^{(2)-1} m_{\nu}^{-1} & W^{-} W^{-} \\
D_{e}^{*} W^{+} & 0 & G_{e}^{*-1}
\end{array}\right)
$$

Here $G_{e}^{(2)}=\phi^{-2} G_{e} \phi^{2}$. This amounts to shifting $D_{e} \rightarrow D_{e}^{(2)}=D_{e}+2 \varphi$, and comes about from $D_{e-} D_{e}=m_{\nu} D_{e-}^{(2)} D_{e}^{(2)} m_{\nu}^{-1}$ in (5.15). Next, we define a new matrix $K_{2}$ by appending to $K_{1}$ a factor on the right

$$
K_{2}=K_{1}\left(\begin{array}{ccc}
1 & 0 & -G_{\nu} D_{\nu+} W^{-} \\
0 & 1 & 0 \\
0 & 0 & 1
\end{array}\right)
$$

Clearly, the determinant of the appended factor is unity, hence Det $K_{2}=\operatorname{Det} K_{1}$, and the effective action remains unchanged. An explicit calculation produces:

$$
K_{2}=\left(\begin{array}{ccc}
G_{\nu}^{-1} & -W^{+} D_{e} & 0 \\
-W^{-} D_{\nu-} & m_{\nu} G_{e}^{(2)}-1 m_{\nu}^{-1} & m_{\nu} W^{-} G_{\nu}^{\prime} W^{-} m_{\nu} \\
D_{e}^{*} W^{+} & 0 & G_{e}^{*-1}-D_{e}^{*} W^{+} G_{\nu} D_{\nu+} W^{-}
\end{array}\right)
$$


where we have introduced a modified neutrino propagator

$$
G_{\nu}^{\prime}:=\left(m_{\nu}^{2}-D_{\nu-}^{(-2)} D_{\nu+}^{(2)}\right)^{-1}
$$

and we have made use of the identity

$$
1+D_{\nu-} G_{\nu} D_{\nu+}=m_{\nu} G_{\nu}^{\prime} m_{\nu}
$$

The sought for form of $K$ with no inverse powers of $m_{\nu}$ is obtained by multiplying $K_{2}$ by $\operatorname{diag}\left(m_{\nu}^{-1}, m_{\nu}^{-1}, 1\right)$ on the left, and its inverse on the right:

$$
K:=\left(\begin{array}{ccc}
G_{\nu}^{(2)-1} & -W^{+} D_{e}^{(2)} & 0 \\
-W^{-} D_{\nu-}^{(2)} & G_{e}^{(2)-1} & W^{-} G_{\nu}^{\prime} W^{-} m_{\nu} \\
D_{e}^{*} W^{+} m_{\nu} & 0 & G_{e}^{*-1}-D_{e}^{*} W^{+} G_{\nu} D_{\nu+} W^{-}
\end{array}\right)
$$

This is our final form of the operator $K$ for Majorana neutrinos. Let us emphasize that $K$ provides the chiral invariant part of the lepton-induced effective action for any number of $W$ 's and for all sectors, $\mathrm{P}$ even or odd and CP even or odd, and its use is not restricted to a derivative expansion.

We note that all manipulations used above contain only blocks with an even number of Dirac matrices, so no problem arises from the presence of the factor $P_{R}$ in the trace in (5.8). The same remark applies for the next section.

\section{Effective action in the CP odd sector}

\section{1 $\Gamma_{4}$ for Majorana and Dirac neutrinos}

The effective action is given by

$$
\Gamma=-\frac{1}{2} \operatorname{Tr}\left(P_{R} \log K\right) .
$$

In order to use this form for the CP odd sector we will expand the right-hand side in powers of $W^{ \pm}$. To this end let us express $K$ in the form

$$
K=K_{0}\left(1-\Delta_{1}-\Delta_{2}\right),
$$

with

$$
\begin{aligned}
K_{0} & =\left(\begin{array}{ccc}
G_{\nu}^{(2)}-1 & & 0 \\
0 & G_{e}^{(2)}-1 & 0 \\
0 & 0 & G_{e}^{*-1}
\end{array}\right), \\
\Delta_{1} & =\left(\begin{array}{ccc}
0 & G_{\nu}^{(2)} W^{+} D_{e}^{(2)} & 0 \\
G_{e}^{(2)} W^{-} D_{\nu-}^{(2)} & 0 & 0 \\
-G_{e}^{*} D_{e}^{*} W^{+} m_{\nu} & 0 & 0
\end{array}\right), \Delta_{2}=\left(\begin{array}{ccc}
0 & 0 & 0 \\
0 & 0 & -G_{e}^{(2)} W^{-} G_{\nu}^{\prime} W^{-} m_{\nu} \\
0 & 0 & G_{e}^{*} D_{e}^{*} W^{+} G_{\nu} D_{\nu+} W^{-}
\end{array}\right) .
\end{aligned}
$$


In this way (once again using formal manipulations which are justified at the order we are working, due to UV convergence)

$$
\operatorname{Tr}\left(P_{R} \log K\right)=\operatorname{Tr}\left(P_{R} \log K_{0}\right)-\sum_{n=1}^{\infty} \frac{1}{n} \operatorname{Tr}\left(P_{R}\left(\Delta_{1}+\Delta_{2}\right)^{n}\right) .
$$

As has been shown in section 4 , the first term that can contribute to $\mathrm{CP}$ violation is of order 4 in powers of $W^{ \pm}$. This selects the terms (with the notation $\left[\Delta_{1}\right]_{1,2}=$ $G_{\nu}^{(2)} W^{+} D_{e}^{(2)}$, etc.)

$$
\begin{aligned}
\operatorname{Tr}\left(P_{R} \log K\right)_{4} & =-\operatorname{Tr}\left(P_{R}\left(\frac{1}{4} \Delta_{1}^{4}+\frac{1}{2} \Delta_{2}^{2}+\Delta_{1}^{2} \Delta_{2}\right)\right) \\
& =-\operatorname{Tr}\left(P_{R}\left(\frac{1}{2}\left(\left[\Delta_{1}\right]_{1,2}\left[\Delta_{1}\right]_{2,1}\right)^{2}+\frac{1}{2}\left(\left[\Delta_{2}\right]_{3,3}\right)^{2}+\left[\Delta_{1}\right]_{1,2}\left[\Delta_{2}\right]_{2,3}\left[\Delta_{1}\right]_{3,1}\right)\right) .
\end{aligned}
$$

Explictly,

$$
\begin{aligned}
\Gamma_{4}= & \frac{1}{2} \operatorname{Tr}\left(P _ { R } \left[\frac{1}{2}\left(G_{e}^{(2)} W^{-} D_{\nu-}^{(2)} G_{\nu}^{(2)} W^{+} D_{e}^{(2)}\right)^{2}+\frac{1}{2}\left(G_{e}^{*} D_{e}^{*} W^{+} G_{\nu} D_{\nu+} W^{-}\right)^{2}\right.\right. \\
& \left.\left.+G_{\nu}^{(2)} W^{+} D_{e}^{(2)} G_{e}^{(2)} W^{-} G_{\nu}^{\prime} m_{\nu} W^{-} G_{e}^{*} D_{e}^{*} W^{+} m_{\nu}\right]\right) .
\end{aligned}
$$

The two terms with factors one half have a similar structure and they are actually equal. This can be shown by applying transposition to the second term, plus the relations

$$
\begin{array}{ll}
\not D_{e}^{*(n) T}=C^{-1} \not D_{e}^{(-n)} C, & D_{\nu \pm}^{(n) T}=C^{-1} \not D_{\nu \mp}^{(-n)} C, \quad W^{ \pm T}=-C^{-1} W^{ \pm} C, \\
G_{e}^{*(n) T}=C^{-1} G_{e}^{(-n)} C, & G_{\nu}^{(n) T}=C^{-1} G_{\nu}^{(-n)} C .
\end{array}
$$

In summary, for Majorana neutrinos, the terms of the effective action with exactly four charged gauge bosons can be expressed as

$$
\Gamma_{4, M}=\frac{1}{2} \operatorname{Tr} P_{R}\left[\left(G_{e} W^{-} \not D_{\nu-} G_{\nu} W^{+} \not D_{e}\right)^{2}+G_{e}^{(2)} W^{-} G_{\nu}^{\prime} m_{\nu} W^{-} G_{e}^{*} \not_{e}^{*} W^{+} G_{\nu} W^{+} \not D_{e} m_{\nu}\right]
$$

The similar expression for Dirac neutrinos is instead

$$
\Gamma_{4, D}=\frac{1}{2} \operatorname{Tr} P_{R}\left[\left(G_{e} W^{-} \not D_{\nu} G_{\nu}^{D} W^{+} \not D_{e}\right)^{2}\right] .
$$

where

$$
G_{\nu}^{D}:=\left(m_{\nu}^{2}-\not D_{\nu+} \not D_{\nu}\right)^{-1}
$$

Here $m_{\nu}$ stands for $(\phi / v) m_{\nu}$ and it is obtained from $M_{D}=A m_{\nu} D$ ( $A$ and $D$ being unitary matrices). $G_{e}$ is the same as before, with $U=B^{\dagger} A^{*}$ and $M_{e}=B m_{e} C$, as in eq. (5.14).

The effective action for Majorana neutrinos contains two types of terms

$$
\Gamma_{4, M}=\Gamma_{4, a}+\Gamma_{4, b}
$$

The terms in $\Gamma_{4, a}$ follow a mechanism similar to that of the Dirac case, namely, the charged bosons alternate along the fermion loop, $W^{-} W^{+} W^{-} W^{+}$(see figure 2, left panel). 
In fact, the expressions of $\Gamma_{4, a}$ and $\Gamma_{4, D}$ are identical for contributions not involving $Z_{\mu}$ nor $\varphi_{\mu}$ and so in this case they give the same contributions. ${ }^{7}$ On the other hand, the mechanism in $\Gamma_{4, b}$ is of the type $W^{-} W^{-} W^{+} W^{+}$(see figure 2, right panel) thereby violating fermionic number conservation. This is the mechanism responsible for neutrinoless double beta decay. The presence of such mechanism is particularly clear in the operator $K$ from the matrix element $[K]_{2,3}=W^{-} G_{\nu}^{\prime} W^{-} m_{\nu}$ in (5.23).

It is also noteworthy that, from CPT invariance one expects that $W^{+}$and $W^{-}$should play similar roles. The symmetry between $W^{+}$and $W^{-}$is explicit in the Dirac operator of eq. (5.1) but it is not manifest in $K$. As noted we could have started from $K_{R}$. In this case the roles of the charged bosons would be reversed. The symmetry is restored in $\Gamma_{4}$ and certainly in the final results.

\subsection{Method of covariant symbols}

The actual calculation of the terms $\Gamma_{4+2}$ has been done using the method of covariant symbols $[62,63,79]$. Quite simply, for any operator of the type $f(D, M)$, where the $D$ are covariant derivatives and the $M(x)$ are matrices in internal space, such as that in eq. (6.8), the functional trace can be expressed as

$$
\operatorname{Tr} f(D, M)=\int \frac{d^{d} x d^{d} p}{(2 \pi)^{d}} \operatorname{tr} f(\bar{D}, \bar{M}) .
$$

tr refers to internal degrees of freedom, $p_{\mu}$ is the fermion loop momentum, and $\bar{D}$ and $\bar{M}$ are the covariant symbols of $D$ and $M$. These are gauge covariant operators which are multiplicative with respect to $x$ and contain derivatives with respect to $p$, namely,

$$
\begin{aligned}
\bar{M} & =\sum_{n=0}^{\infty} \frac{i^{n}}{n !}\left(\hat{D}_{\alpha_{1}} \cdots \hat{D}_{\alpha_{n}} M\right) \partial_{\alpha_{1}}^{p} \cdots \partial_{\alpha_{n}}^{p} \\
\bar{D}_{\mu} & =i p_{\mu}+\sum_{n=1}^{\infty} \frac{i^{n} n}{(n+1) !}\left(\hat{D}_{\alpha_{1}} \cdots \hat{D}_{\alpha_{n}} D_{\mu}\right) \partial_{\alpha_{1}}^{p} \cdots \partial_{\alpha_{n}}^{p}
\end{aligned}
$$

with $\partial_{\alpha}^{p}=\partial / \partial p_{\alpha}$ and $\hat{D}_{\alpha} X=\left[D_{\alpha}, X\right]$, in particular, $\hat{D}_{\alpha} D_{\mu}=F_{\alpha \mu}$. The crucial property of the covariant symbols, besides being manifestly gauge covariant and multiplicative as operators, is that they define a representation of the algebra of operators, that is, $\overline{f(X, Y)}=$ $f(\bar{X}, \bar{Y})$. Therefore, one can simply compute the symbols of the basic blocks and use them in the full expression.

In our case an application of the method of covariant symbols in (6.8) produces

$$
\begin{aligned}
\Gamma_{4, a} & =\int \frac{d^{4} x d^{4} p}{(2 \pi)^{4}} \frac{1}{2} \operatorname{tr}\left[P_{R}\left(\bar{G}_{e} \bar{W}^{-} \bar{D}_{\nu-} \bar{G}_{\nu} \bar{W}^{+} \overline{D D}_{e}\right)^{2}\right] \\
\Gamma_{4, b} & =\int \frac{d^{4} x d^{4} p}{(2 \pi)^{4}} \frac{1}{2} \operatorname{tr}\left[P_{R} \bar{G}_{e}^{(2)} \bar{W}^{-} \bar{G}_{\nu}^{\prime} \bar{m}_{\nu} \bar{W}^{-} \bar{G}_{e}^{*} \bar{D}_{e}^{*} \bar{W}^{+} \bar{G}_{\nu} \bar{W}^{+} \overline{D D}_{e} \bar{m}_{\nu}\right],
\end{aligned}
$$

${ }^{7}$ This refers to the explicit $Z_{\mu}$. The $Z^{0}$ field appears also in $D_{e}$ together with the photon field, eq. (3.5). Unfortunately, the Dirac results in [51] cannot be directly adapted to $\Gamma_{4, a}$ when Higgs or $Z^{0}$ are present by means of some clever redefinition of the fields there. 
where tr refers to Dirac and flavor spaces. The covariant symbols of the basics operators in (6.14) are displayed in appendix A.

We want to work out the leading $\mathrm{CP}$ violating terms, that is, those driven by operators of lowest dimension, which are those in $\Gamma_{4+2}$. Therefore, in (6.14) we select contributions with exactly two derivatives, where each $\hat{D}_{\mu}, Z_{\mu}$ or $\varphi_{\mu}$ counts as one derivative.

Using the covariant symbols in appendix A, the calculation of the effective action proceeds from (6.14) by i) removing all momentum derivatives, applying them either on the right or the left, ii) taking an angular average over the momenta, iii) evaluating the Dirac gamma traces, iv) factoring each term into a momentum integral (involving the mass matrices) and an operator (involving the external fields and their derivatives), v) rearranging indices in the operators, including Bianchi identities (namely, $\left[\hat{D}_{\alpha}, \hat{D}_{\beta}\right] X=$ $\left.\left[F_{\alpha \beta}, X\right]\right)$, vi) using integration by parts, and vii) using identities between momentum integrals to simplify the final result. At step iv) the $\mathrm{CP}$ odd terms can be already isolated by selecting the antihermitian/hermitian part of the operators in the $\mathrm{P}$ even/odd sectors. The calculation has been repeated using the method of ordinary symbols $[80,81]$ as a check of the results.

\subsection{Allowed operators and their couplings}

To express the results we introduce two bases of CP odd operators, one with parity even operators and another with parity odd ones. They are displayed in tables 2 and 3 . In the operators of the parity odd sector, the labels $a$ and $s$ denote antisymmetric and symmetric Lorentz indices, respectively. So for instance

$$
W_{a}^{+} W_{s}^{+} W_{a}^{-} W_{s}^{-} Z_{a} \varphi_{a} \equiv \epsilon_{\mu \nu \alpha \beta} W_{\mu}^{+} W_{\rho}^{+} W_{\nu}^{-} W_{\rho}^{-} Z_{\alpha} \varphi_{\beta} .
$$

Operators with $W^{ \pm}$carrying more than one derivative have been excluded from the bases, as those operators can be eliminated through integration by parts.

Identities from integration by parts exist among the operators. To establish such relations, one should take into account that the Higgs field $\phi$ is present in the momentum integrals, through the mass terms, and this may produce new $\varphi_{\mu}$ dependences not explicit in eq. (6.8),

$$
0=\int d^{4} x \operatorname{tr} \hat{D}_{\mu}\left(I \mathcal{O}_{\mu}\right)=\int d^{4} x \operatorname{tr}\left(\varphi_{\mu} \phi \frac{\partial I}{\partial \phi} \mathcal{O}_{\mu}+I \hat{D}_{\mu} \mathcal{O}_{\mu}\right)
$$

To make things simpler, we have assumed that the coupling between mass and Higgs is predominantly of the type $m_{i} \rightarrow \phi m_{i}$ (Dirac particles). In this case $\phi(\partial I / \partial \phi)=-2 I$ for operators of dimension 6 . It should not go unnoticed that this is an approximation taken on otherwise exact relations, imposed on us by the need to avoid cumbersome expressions. It only affects the Majorana neutrino case in terms with $\varphi_{\mu}$. 


\begin{tabular}{|c|c|c|c|}
\hline$A_{1}^{+}$ & $W_{\alpha}^{+} W_{\alpha}^{+} W_{\beta \beta}^{-} W_{\gamma \gamma}^{-}-$c.c. & $A_{21}^{+}$ & $W_{\alpha}^{+} W_{\beta}^{+} W_{\alpha}^{-} W_{\beta}^{-} Z_{\gamma \gamma}$ \\
\hline$A_{2}^{+}$ & $W_{\alpha}^{+} W_{\alpha}^{+} W_{\beta \gamma}^{-} W_{\beta \gamma}^{-}-$c.c. & $A_{22}^{+}$ & $W_{\alpha}^{+} W_{\beta}^{+} W_{\alpha}^{-} W_{\gamma}^{-} Z_{\beta \gamma}-$ c.c. \\
\hline$A_{3}^{+}$ & $W_{\alpha}^{+} W_{\alpha}^{+} W_{\beta \gamma}^{-} W_{\gamma \beta}^{-}-$c.c. & $A_{23}^{+}$ & $W_{\alpha \beta}^{+} W_{\gamma}^{+} W_{\alpha}^{-} W_{\beta}^{-} Z_{\gamma}-$ c.c. \\
\hline$A_{4}^{+}$ & $W_{\alpha}^{+} W_{\beta}^{+} W_{\alpha \beta}^{-} W_{\gamma \gamma}^{-}-$c.c. & $A_{24}^{+}$ & $W_{\alpha}^{+} W_{\beta}^{+} W_{\alpha \beta}^{-} W_{\gamma}^{-} \varphi_{\gamma}-$ c.c. \\
\hline$A_{5}^{+}$ & $W_{\alpha}^{+} W_{\beta}^{+} W_{\alpha \gamma}^{-} W_{\beta \gamma}^{-}-$c.c. & $A_{25}^{+}$ & $W_{\alpha \beta}^{+} W_{\gamma}^{+} W_{\alpha}^{-} W_{\gamma}^{-} Z_{\beta}-$ c.c. \\
\hline$A_{6}^{+}$ & $W_{\alpha}^{+} W_{\beta}^{+} W_{\alpha \gamma}^{-} W_{\gamma \beta}^{-}-$c.c. & $A_{26}^{+}$ & $W_{\alpha}^{+} W_{\beta}^{+} W_{\alpha \gamma}^{-} W_{\beta}^{-} \varphi_{\gamma}-$ c.c. \\
\hline$A_{7}^{+}$ & $W_{\alpha}^{+} W_{\beta}^{+} W_{\gamma \alpha}^{-} W_{\gamma \beta}^{-}-$c.c. & $A_{27}^{+}$ & $W_{\alpha \beta}^{+} W_{\beta}^{+} W_{\alpha}^{-} W_{\gamma}^{-} Z_{\gamma}-$ c.c. \\
\hline$A_{8}^{+}$ & $W_{\alpha \alpha}^{+} W_{\beta}^{+} W_{\beta \gamma}^{-} W_{\gamma}^{-}-$c.c. & $A_{28}^{+}$ & $W_{\alpha}^{+} W_{\beta}^{+} W_{\alpha \gamma}^{-} W_{\gamma}^{-} \varphi_{\beta}-$ c.c. \\
\hline$A_{9}^{+}$ & $W_{\alpha \alpha}^{+} W_{\beta}^{+} W_{\gamma \beta}^{-} W_{\gamma}^{-}-$c.c. & $A_{29}^{+}$ & $W_{\alpha \beta}^{+} W_{\gamma}^{+} W_{\beta}^{-} W_{\gamma}^{-} Z_{\alpha}-$ c.c. \\
\hline$A_{10}^{+}$ & $W_{\alpha \beta}^{+} W_{\alpha}^{+} W_{\beta \gamma}^{-} W_{\gamma}^{-}-$c.c. & $A_{30}^{+}$ & $W_{\alpha}^{+} W_{\beta}^{+} W_{\gamma \alpha}^{-} W_{\beta}^{-} \varphi_{\gamma}-$ c.c. \\
\hline$A_{11}^{+}$ & $W_{\alpha \beta}^{+} W_{\gamma}^{+} W_{\beta \gamma}^{-} W_{\alpha}^{-}-$c.c. & $A_{31}^{+}$ & $W_{\alpha \beta}^{+} W_{\alpha}^{+} W_{\beta}^{-} W_{\gamma}^{-} Z_{\gamma}-$ c.c. \\
\hline$A_{12}^{+}$ & $W_{\alpha}^{+} W_{\alpha}^{+} W_{\beta}^{-} W_{\beta}^{-} Z_{\gamma \gamma}$ & $A_{32}^{+}$ & $W_{\alpha}^{+} W_{\beta}^{+} W_{\gamma \alpha}^{-} W_{\gamma}^{-} \varphi_{\beta}-$ c.c. \\
\hline$A_{13}^{+}$ & $W_{\alpha}^{+} W_{\alpha}^{+} W_{\beta}^{-} W_{\gamma}^{-} Z_{\beta \gamma}-$ c.c. & $A_{33}^{+}$ & $W_{\alpha \alpha}^{+} W_{\beta}^{+} W_{\beta}^{-} W_{\gamma}^{-} Z_{\gamma}-$ c.c. \\
\hline$A_{14}^{+}$ & $W_{\alpha}^{+} W_{\alpha}^{+} W_{\beta}^{-} W_{\gamma}^{-} \varphi_{\beta \gamma}-$ c.c. & $A_{34}^{+}$ & $W_{\alpha}^{+} W_{\beta}^{+} W_{\gamma \gamma}^{-} W_{\alpha}^{-} \varphi_{\beta}-$ c.c. \\
\hline$A_{15}^{+}$ & $W_{\alpha \alpha}^{+} W_{\beta}^{+} W_{\gamma}^{-} W_{\gamma}^{-} Z_{\beta}-$ c.c. & $A_{35}^{+}$ & $W_{\alpha}^{+} W_{\alpha}^{+} W_{\beta}^{-} W_{\beta}^{-} Z_{\gamma} \varphi_{\gamma}$ \\
\hline$A_{16}^{+}$ & $W_{\alpha}^{+} W_{\alpha}^{+} W_{\beta \beta}^{-} W_{\gamma}^{-} \varphi_{\gamma}-$ c.c. & $A_{36}^{+}$ & $W_{\alpha}^{+} W_{\alpha}^{+} W_{\beta}^{-} W_{\gamma}^{-} Z_{\beta} Z_{\gamma}-$ c.c. \\
\hline$A_{17}^{+}$ & $W_{\alpha \beta}^{+} W_{\alpha}^{+} W_{\gamma}^{-} W_{\gamma}^{-} Z_{\beta}-$ c.c. & $A_{37}^{+}$ & $W_{\alpha}^{+} W_{\alpha}^{+} W_{\beta}^{-} W_{\gamma}^{-} Z_{\beta} \varphi_{\gamma}-$ c.c. \\
\hline$A_{18}^{+}$ & $W_{\alpha}^{+} W_{\alpha}^{+} W_{\beta \gamma}^{-} W_{\beta}^{-} \varphi_{\gamma}-$ c.c. & $A_{38}^{+}$ & $W_{\alpha}^{+} W_{\alpha}^{+} W_{\beta}^{-} W_{\gamma}^{-} \varphi_{\beta} \varphi_{\gamma}-$ c.c. \\
\hline$A_{19}^{+}$ & $W_{\alpha \beta}^{+} W_{\beta}^{+} W_{\gamma}^{-} W_{\gamma}^{-} Z_{\alpha}-$ c.c. & $A_{39}^{+}$ & $W_{\alpha}^{+} W_{\beta}^{+} W_{\alpha}^{-} W_{\beta}^{-} Z_{\gamma} \varphi_{\gamma}$ \\
\hline$A_{20}^{+}$ & $W_{\alpha}^{+} W_{\alpha}^{+} W_{\beta \gamma}^{-} W_{\gamma}^{-} \varphi_{\beta}-$ c.c. & $A_{40}^{+}$ & $W_{\alpha}^{+} W_{\beta}^{+} W_{\alpha}^{-} W_{\gamma}^{-} Z_{\beta} \varphi_{\gamma}-$ c.c. \\
\hline
\end{tabular}

Table 2. List of $\mathrm{P}$ even and CP odd operators of the type $4+2$.

\begin{tabular}{|ll|cl|}
\hline$A_{1}^{-}$ & $W_{a}^{+} W_{a s}^{+} W_{a}^{-} W_{a s}^{-}$ & $A_{14}^{-}$ & $W_{a}^{+} W_{s}^{+} W_{a}^{-} W_{s}^{-} Z_{a a}$ \\
$A_{2}^{-}$ & $W_{a}^{+} W_{a s}^{+} W_{a}^{-} W_{s a}^{-}+$c.c. & $A_{15}^{-}$ & $W_{a}^{+} W_{a a}^{+} W_{a}^{-} W_{s}^{-} Z_{s}+$ c.c. \\
$A_{3}^{-}$ & $W_{a}^{+} W_{s a}^{+} W_{a}^{-} W_{s a}^{-}$ & $A_{16}^{-}$ & $W_{a}^{+} W_{s}^{+} W_{a}^{-} W_{a a}^{-} \varphi_{s}+$ c.c. \\
$A_{4}^{-}$ & $W_{a}^{+} W_{a a}^{+} W_{a}^{-} W_{s s}^{-}+$c.c. & $A_{17}^{-}$ & $W_{a}^{+} W_{s}^{+} W_{a}^{-} W_{a s}^{-} Z_{a}+$ c.c. \\
$A_{5}^{-}$ & $W_{a}^{+} W_{a s}^{+} W_{s}^{-} W_{a a}^{-}+$c.c. & $A_{18}^{-}$ & $W_{a}^{+} W_{a s}^{+} W_{a}^{-} W_{s}^{-} \varphi_{a}+$ c.c. \\
$A_{6}^{-}$ & $W_{a}^{+} W_{s a}^{+} W_{s}^{-} W_{a a}^{-}+$c.c. & $A_{19}^{-}$ & $W_{a}^{+} W_{s}^{+} W_{a}^{-} W_{s a}^{-} Z_{a}+$ c.c. \\
$A_{7}^{-}$ & $W_{s}^{+} W_{a a}^{+} W_{s}^{-} W_{a a}^{-}$ & $A_{20}^{-}$ & $W_{a}^{+} W_{s a}^{+} W_{a}^{-} W_{s}^{-} \varphi_{a}+$ c.c. \\
$A_{8}^{-}$ & $W_{a}^{+} W_{a a}^{+} W_{s}^{-} W_{a s}^{-}+$c.c. & $A_{21}^{-}$ & $W_{a}^{+} W_{a a}^{+} W_{s}^{-} W_{s}^{-} Z_{a}+$ c.c. \\
$A_{9}^{-}$ & $W_{a}^{+} W_{a a}^{+} W_{s}^{-} W_{s a}^{-}+$c.c. & $A_{22}^{-}$ & $W_{a}^{+} W_{a a}^{+} W_{s}^{-} W_{s}^{-} \varphi_{a}+$ c.c. \\
$A_{10}^{-}$ & $W_{a a}^{+} W_{a a}^{+} W_{s}^{-} W_{s}^{-}+$c.c. & $A_{23}^{-}$ & $W_{a}^{+} W_{s}^{+} W_{s}^{-} W_{a a}^{-} Z_{a}+$ c.c. \\
$A_{11}^{-}$ & $W_{a}^{+} W_{s}^{+} W_{a a}^{-} W_{a s}^{-}+$c.c. & $A_{24}^{-}$ & $W_{a}^{+} W_{s}^{+} W_{s}^{-} W_{a a}^{-} \varphi_{a}+$ c.c. \\
$A_{12}^{-}$ & $W_{a}^{+} W_{s}^{+} W_{a a}^{-} W_{s a}^{-}+$c.c. & $A_{25}^{-}$ & $W_{a}^{+} W_{s}^{+} W_{a}^{-} W_{s}^{-} Z_{a} \varphi_{a}$ \\
$A_{13}^{-}$ & $F_{a a}^{e} W_{a}^{+} W_{s}^{+} W_{a}^{-} W_{s}^{-}$ & &
\end{tabular}

Table 3. List of P odd and CP odd operators of the type $4+2$. 
With this proviso, the following by-parts integration relations are found among the $\mathrm{P}$ even operators

$$
\begin{aligned}
& 0=A_{1}^{+}-A_{3}^{+}-2 A_{8}^{+}+2 A_{10}^{+}-2 A_{16}^{+}+2 A_{18}^{+}, \\
& 0=A_{4}^{+}-A_{6}^{+}-A_{9}^{+}+A_{11}^{+}+2 A_{26}^{+}-2 A_{34}^{+}, \\
& 0=A_{24}^{+}+A_{26}^{+}-A_{32}^{+}-A_{34}^{+}, \\
& 0=A_{12}^{+}+2 A_{19}^{+}-2 A_{35}^{+}, \\
& 0=A_{13}^{+}+A_{15}^{+}+A_{17}^{+}+2 A_{27}^{+}-2 A_{37}^{+}, \\
& 0=A_{14}^{+}+A_{16}^{+}+A_{18}^{+}-2 A_{28}^{+}-2 A_{38}^{+}, \\
& 0=A_{21}^{+}+2 A_{29}^{+}-2 A_{39}^{+}, \\
& 0=A_{22}^{+}+A_{23}^{+}+A_{25}^{+}+A_{31}^{+}+A_{33}^{+}-2 A_{40}^{+} .
\end{aligned}
$$

Likewise, for the $\mathrm{P}$ odd operators one finds the relations

$$
\begin{aligned}
& 0=2 A_{1}^{-}-A_{5}^{-}+A_{11}^{-}-A_{13}^{-}-2 A_{18}^{-} \\
& 0=2 A_{1}^{-}-A_{2}^{-}+A_{4}^{-}+A_{5}^{-}-\frac{1}{2} A_{10}^{-}-A_{22}^{-} \\
& 0=A_{5}^{-}-2 A_{7}^{-}+A_{11}^{-}+A_{13}^{-}-2 A_{24}^{-} \\
& 0=A_{14}^{-}+A_{17}^{-}-A_{23}^{-}+2 A_{25}^{-} .
\end{aligned}
$$

In addition, the $\mathrm{P}$ odd operators are not independent due to the four-dimensional identity

$$
X_{\mu, a, a, a, a}-X_{a, \mu, a, a, a}+X_{a, a, \mu, a, a}-X_{a, a, a, \mu, a}+X_{a, a, a, a, \mu}=0 .
$$

As a consequence, the following relations exist

$$
\begin{aligned}
& 0=-A_{1}^{-}+A_{2}^{-}-A_{3}^{-}-A_{5}^{-}+A_{6}^{-}-A_{7}^{-}, \\
& 0=-2 A_{1}^{-}+A_{2}^{-}-A_{4}^{-}-A_{5}^{-}+A_{8}^{-}, \\
& 0=-A_{1}^{-}+A_{3}^{-}-A_{4}^{-}-A_{5}^{-}-A_{7}^{-}+A_{9}^{-}, \\
& 0=-\frac{1}{2} A_{10}^{-}-A_{11}^{-}+A_{12}^{-}, \\
& 0=A_{15}^{-}-A_{17}^{-}+A_{19}^{-}-A_{21}^{-}-A_{23}^{-}, \\
& 0=A_{16}^{-}-A_{18}^{-}+A_{20}^{-}+A_{22}^{-}-A_{24}^{-} .
\end{aligned}
$$

The effective actions $\Gamma_{4+2, a}^{ \pm}$and $\Gamma_{4+2, b}^{ \pm}$for Majorana neutrinos and $\Gamma_{4+2, D}^{ \pm}$for Dirac ones can be expressed in the form

$$
\begin{aligned}
\Gamma_{4+2, a}^{+} & =\int d^{4} x \frac{v^{2}}{\phi^{2}} \sum_{k} i g_{a, k}^{+} A_{k}^{+}, & \Gamma_{4+2, a}^{-} & =\int d^{4} x \frac{v^{2}}{\phi^{2}} \sum_{k} i g_{a, k}^{-} A_{k}^{-}, \\
\Gamma_{4+2, b}^{+} & =\int d^{4} x \frac{v^{2}}{\phi^{2}} \sum_{k} i g_{b, k}^{+} A_{k}^{+}, & \Gamma_{4+2, b}^{-} & =\int d^{4} x \frac{v^{2}}{\phi^{2}} \sum_{k} i g_{b, k}^{-} A_{k}^{-}, \\
\Gamma_{4+2, D}^{+} & =\int d^{4} x \frac{v^{2}}{\phi^{2}} \sum_{k} i g_{D, k}^{+} A_{k}^{+}, & \Gamma_{4+2, D}^{-} & =0 .
\end{aligned}
$$




\begin{tabular}{|rr|cr|cr|cr|}
\hline$g_{a, 1}^{+}$ & $-\frac{1}{4} a_{1}$ & $g_{a, 10}^{+}$ & $-\frac{1}{6} a_{1}$ & $g_{a, 20}^{+}$ & $\frac{1}{2} a_{1}-\frac{5}{3} a_{2}$ & $g_{a, 33}^{+}$ & $-2 a_{1}$ \\
$g_{a, 2}^{+}$ & $\frac{5}{6} a_{1}$ & $g_{a, 11}^{+}$ & $-\frac{2}{3} a_{1}$ & $g_{a, 22}^{+}$ & $-\frac{2}{3} a_{1}$ & $g_{a, 34}^{+}$ & $\frac{1}{3} a_{1}-\frac{2}{3} a_{2}$ \\
$g_{a, 3}^{+}$ & $-\frac{1}{12} a_{1}$ & $g_{a, 12}^{+}$ & $\frac{1}{6} a_{1}$ & $g_{a, 24}^{+}$ & $a_{1}-\frac{2}{3} a_{2}$ & $g_{a, 35}^{+}$ & $\frac{20}{3} a_{1}+\frac{2}{3} a_{2}$ \\
$g_{a, 5}^{+}$ & $-\frac{1}{3} a_{1}$ & $g_{a, 13}^{+}$ & $\frac{1}{3} a_{1}$ & $g_{a, 26}^{+}$ & $-\frac{1}{3} a_{1}-\frac{2}{3} a_{2}$ & $g_{a, 36}^{+}$ & $4 a_{1}$ \\
$g_{a, 6}^{+}$ & $\frac{4}{3} a_{1}$ & $g_{a, 15}^{+}$ & $2 a_{1}$ & $g_{a, 28}^{+}$ & $-a_{1}+\frac{2}{3} a_{2}$ & $g_{a, 37}^{+}$ & $\frac{4}{3} a_{2}$ \\
$g_{a, 7}^{+}$ & $-a_{1}$ & $g_{a, 16}^{+}$ & $\frac{5}{3} a_{1}+\frac{1}{3} a_{2}$ & $g_{a, 30}^{+}$ & $-a_{1}+2 a_{2}$ & $g_{a, 38}^{+}$ & $4 a_{1}$ \\
$g_{a, 8}^{+}$ & $\frac{1}{6} a_{1}$ & $g_{a, 17}^{+}$ & $-2 a_{1}$ & $g_{a, 31}^{+}$ & $2 a_{1}$ & $g_{a, 39}^{+}$ & $-\frac{8}{3} a_{1}$ \\
$g_{a, 9}^{+}$ & $\frac{2}{3} a_{1}$ & $g_{a, 18}^{+}$ & $-\frac{8}{3} a_{1}+\frac{1}{3} a_{2}$ & $g_{a, 32}^{+}$ & $3 a_{1}-\frac{2}{3} a_{2}$ & $g_{a, 40}^{+}$ & $-\frac{8}{3} a_{2}$ \\
\hline
\end{tabular}

Table 4. Non vanishing couplings for $\Gamma_{4+2, a}^{+}$.

\begin{tabular}{|lr|cr|cr|}
\hline$g_{b, 2}^{+}$ & $-b_{1}+b_{2}$ & $g_{b, 16}^{+}$ & $-\frac{5}{2} b_{1}+4 b_{2}$ & $g_{b, 28}^{+}$ & $4 b_{1}$ \\
$g_{b, 8}^{+}$ & $b_{1}$ & $g_{b, 17}^{+}$ & $\frac{7}{2} b_{1}-3 b_{2}$ & $g_{b, 35}^{+}$ & $10 b_{1}-15 b_{3}+2 b_{4}-b_{5}$ \\
$g_{b, 10}^{+}$ & $-1 b_{1}$ & $g_{b, 18}^{+}$ & $\frac{5}{2} b_{1}-4 b_{2}$ & $g_{b, 36}^{+}$ & $-4 b_{3}$ \\
$g_{b, 12}^{+}$ & $\frac{3}{4} b_{1}+\frac{1}{2} b_{2}$ & $g_{b, 20}^{+}$ & $\frac{13}{2} b_{1}-4 b_{2}-2 b_{4}+b_{5}$ & $g_{b, 37}^{+}$ & $-8 b_{1}+12 b_{2}$ \\
$g_{b, 15}^{+}$ & $-\frac{7}{2} b_{1}+3 b_{2}$ & $g_{b, 27}^{+}$ & $-4 b_{1}$ & $g_{b, 38}^{+}$ & $-2 b_{1}+8 b_{2}$ \\
\hline
\end{tabular}

Table 5. Non vanishing couplings for $\Gamma_{4+2, b}^{+}$.

\begin{tabular}{|rr|cr|cr|cr|}
\hline$g_{a, 14}^{-}$ & $\frac{2}{3} a_{1}$ & $g_{a, 16}^{-}$ & $2 a_{1}$ & $g_{a, 21}^{-}$ & $-\frac{1}{3} a_{1}$ & $g_{a, 24}^{-}$ & $-2 a_{1}$ \\
$g_{a, 15}^{-}$ & $-\frac{2}{3} a_{1}$ & $g_{a, 18}^{-}$ & $-\frac{2}{3} a_{1}$ & $g_{a, 22}^{-}$ & $\frac{5}{3} a_{1}$ & $g_{a, 25}^{-}$ & $-\frac{8}{3} a_{2}$ \\
\hline
\end{tabular}

Table 6. Non vanishing couplings for $\Gamma_{4+2, a}^{-}$.

\begin{tabular}{|ll|ll|ll|}
\hline$g_{b, 10}^{-}$ & $\frac{1}{2} b_{1}$ & $g_{b, 21}^{-}$ & $\frac{1}{2} b_{1}-b_{2}$ & $g_{b, 22}^{-}$ & $-\frac{1}{2} b_{1}$ \\
\hline
\end{tabular}

Table 7. Non vanishing couplings for $\Gamma_{4+2, b}^{-}$.

The explicit imaginary unit has been introduced so that the couplings $g_{t, k}^{ \pm}$are all real. The operators $A_{k}^{ \pm}$themselves are common for the two structures $\Gamma_{a}$ and $\Gamma_{b}$, but the couplings are sensitive to this structure.

The non vanishing couplings are collected in tables $4,5,6,7$, and 8 . The couplings are expressed in terms of a few independent momentum integrals (defined in (4.8)), namely,

$$
\begin{aligned}
& a_{1} \equiv I_{a, 1,1,2,2}^{6}, \quad a_{2} \equiv I_{a, 1,1,2,3}^{8}, \quad b_{1} \equiv I_{b, 1,1,1,2}^{2}, \quad b_{2} \equiv I_{b, 1,1,1,3}^{4}, \\
& b_{3} \equiv I_{b, 1,1,2,2}^{4}, \quad b_{4} \equiv I_{b, 1,1,3,2}^{6}, \quad b_{5} \equiv I_{b, 2,1,2,2}^{6} \text {. }
\end{aligned}
$$

In eq. (6.21) we have extracted the main dependence on (underivated) $\phi$ from the couplings assuming a Dirac-type Higgs coupling in mass terms. Therefore, in the various momentum integrals above $m_{e}$ no longer contains the factor $(\phi / v)$ and $m_{\nu}$ contains a single factor $(\phi / v)$ in the Majorana case and none in the Dirac case. 


\begin{tabular}{|rr|rr|cr|rr|}
\hline$g_{D, 1}^{+}$ & $-\frac{1}{6} a_{1}$ & $g_{D, 15}^{+}$ & $\frac{4}{3} a_{1}$ & $g_{D, 30}^{+}$ & $\frac{4}{3} a_{1}$ & $g_{D, 37}^{+}$ & $\frac{4}{3} a_{1}$ \\
$g_{D, 2}^{+}$ & $\frac{5}{6} a_{1}$ & $g_{D, 16}^{+}$ & $\frac{4}{3} a_{1}$ & $g_{D, 31}^{+}$ & $\frac{4}{3} a_{1}$ & $g_{D, 38}^{+}$ & $2 a_{1}$ \\
$g_{D, 3}^{+}$ & $-\frac{1}{6} a_{1}$ & $g_{D, 17}^{+}$ & $-\frac{4}{3} a_{1}$ & $g_{D, 32}^{+}$ & $\frac{4}{3} a_{1}$ & $g_{D, 39}^{+}$ & $-\frac{8}{3} a_{1}$ \\
$g_{D, 4}^{+}$ & $\frac{2}{3} a_{1}$ & $g_{D, 18}^{+}$ & $-\frac{4}{3} a_{1}$ & $g_{D, 33}^{+}$ & $-\frac{4}{3} a_{1}$ & $g_{D, 40}^{+}$ & $-\frac{8}{3} a_{1}$ \\
$g_{D, 5}^{+}$ & $-\frac{1}{3} a_{1}$ & $g_{D, 19}^{+}$ & $-\frac{4}{3} a_{1}$ & $g_{D, 34}^{+}$ & $-\frac{4}{3} a_{1}$ & & \\
$g_{D, 6}^{+}$ & $\frac{2}{3} a_{1}$ & $g_{D, 20}^{+}$ & $-\frac{4}{3} a_{1}$ & $g_{D, 35}^{+}$ & $\frac{16}{3} a_{1}$ & & \\
$g_{D, 7}^{+}$ & $-a_{1}$ & $g_{D, 29}^{+}$ & $\frac{4}{3} a_{1}$ & $g_{D, 36}^{+}$ & $2 a_{1}$ & & \\
\hline
\end{tabular}

Table 8. Non vanishing couplings for $\Gamma_{4+2, D}^{+}$.

It should be noted that not all the integrals $I_{a}$ and $I_{b}$ are independent. The following relations have been used to simplify the expressions:

$$
\begin{aligned}
& 5 I_{a, 1,1,2,2}^{6}=2 I_{a, 1,1,2,3}^{8}+2 I_{a, 1,1,3,2}^{8}, \\
& 3 I_{b, 1,1,1,2}^{2}=2 I_{b, 1,1,1,3}^{4}+2 I_{b, 1,1,2,2}^{4}, \\
& 4 I_{b, 1,1,2,2}^{4}=2 I_{b, 1,1,2,3}^{6}+2 I_{b, 1,1,3,2}^{6}+I_{b, 2,1,2,2}^{6}, \\
& 4 I_{b, 1,1,1,3}^{4}=3 I_{b, 1,1,1,4}^{6}+2 I_{b, 1,1,2,3}^{6}+I_{b, 1,2,1,3}^{6} .
\end{aligned}
$$

These relations follow from integration by parts in momentum space and the symmetry or antisymmetry properties of $I_{a}$ and $I_{b}$ (more detailed information, such as $U$ being a unitary matrix in $N_{e}$, is not required).

\subsection{Discussion of the analytical results}

The expressions given for the effective action are in Euclidean space. With the conventions of [51], the expressions in Minkowskian space take exactly the same form except for the two following modifications: $\epsilon_{\mu \nu \alpha \beta} \rightarrow i \epsilon_{\mu \nu \alpha \beta}$ (in $\Gamma^{-}$) and $Z_{\mu} \rightarrow i Z_{\mu}$ (as well as $Z_{\mu \nu} \rightarrow i Z_{\mu \nu}$, etc). The meaning of the symbols changes to conform to the Minkowskian conventions, and so $\left(W^{+}\right)_{\mu}^{*}=W_{\mu}^{-}$and $Z_{\mu}, \varphi_{\mu}$ and $F_{\mu \nu}^{e}$ are real. The resulting real-time effective action is real, both for the parity even and odd components. The true effective action (in Minkowski space) has an imaginary part when the vertex functions contained in it are above the unitarity thresholds. In our calculation we are always below thresholds since the derivative expansion is an expansion around zero four-momentum.

The set of operators is common to the effective actions obtained from integration of leptons or from integration of quarks. For each operator, the total coupling is obtained by adding the quark and lepton contributions. Also, the operators do not distinguish between the couplings of type $I_{a}$, which corresponds to alternating charged bosons along the loop, $W^{+} W^{-} W^{+} W^{-}$, and those of type $I_{b}$, with structure $W^{+} W^{+} W^{-} W^{-}$, which is exclusive for Majorana neutrinos. The total coupling to a given operator is obtained by adding its type $I_{a}$ and type $I_{b}$ contributions. Integrals of the two types $I_{a}$ and $I_{b}$ appear in parity even and parity odd operators.

For Dirac neutrinos, as for quarks, all the couplings to $\mathrm{P}$ odd operators vanish in the $\mathrm{CP}$ sector. The single more interesting result found here is that Majorana neutrinos would 
produce $\mathrm{C}$ even $\mathrm{P}$ odd terms in $\Gamma_{4+2}$. This can be obtained without recourse to $Z$ or Higgs fields, namely,

$$
\frac{1}{2} i b_{1} \epsilon_{\mu \nu \alpha \beta}\left(W_{\mu \nu}^{+} W_{\alpha \beta}^{+} W_{\rho}^{-} W_{\rho}^{-}+\text {c.c. }\right) .
$$

This term corresponds to $A_{10}^{-}$and the coupling is purely of Majorana type $\left(I_{b}\right)$. Further operators of types $I_{a}$ and $I_{b}$ are allowed if $Z$ and $\varphi$ are included. ${ }^{8}$ It is also interesting that this term can be used to show that $\mathrm{CP}$ would be violated, for generic values of $U$ and the masses, in a two generations scenario. Of course, the same would not be true for Dirac neutrinos or quarks, which require at least three generations.

An inspection of the results shows that $F_{\mu \nu}^{e}$ is not present. For $\mathrm{P}$ even terms this follows from the fact that the only $4+2$ operator one can write involving $F_{\mu \nu}^{e}$ is $F_{\mu \nu}^{e} W_{\mu}^{+} W_{\alpha}^{+} W_{\nu}^{-} W_{\alpha}^{-}$, which is $\mathrm{CP}$ even. On the other hand, in the $\mathrm{P}$ odd sector the unique operator with $F_{\mu \nu}^{e}$ is $A_{13}^{-}$and it is $\mathrm{CP}$ odd, however, it can be eliminated using integration by parts.

The effective action for Dirac neutrinos is formally identical to that obtained for quarks in [51]. The calculation there was carried out for generic gauge connections $A_{\mu}^{u}$ and $A_{\mu}^{d}$ (which in the Standard Model take the values $A_{u}=-(2 / 3) A_{e}$ and $A_{d}=+(1 / 3) A_{e}$ ), and so it includes the leptonic case for Dirac neutrinos by setting $A_{u}=0 A_{d}=A_{e}$. Since $F_{\mu \nu}^{u}, F_{\mu \nu}^{d}$ do not appear in the formulas, the results are formally equal. Moreover, the derivatives of the charged gauge fields are also equal since $A_{d}-A_{u}=A_{e}$. As said the final operators are common to leptons and quarks. The difference between the $\mathrm{CP}$ violating effective actions induced by quarks and Dirac leptons comes only from the difference in the mass matrices (i.e., $U$ and masses). We dwell on this in section 7.4.

For Majorana neutrinos, the term $\Gamma_{4+2, b}^{ \pm}$is new and represents a different mechanism which involves (virtual) lepton-number violation. The terms $\Gamma_{4+2, a}^{ \pm}$use the same Kobayashi-Maskawa mechanism as quarks or Dirac neutrinos. As already noticed $\Gamma_{4+2, a}$ coincides with $\Gamma_{4+2, D}$ modulo terms involving $Z$ and Higgs. The explicit calculation shows that they differ in terms depending on $Z$ or $\varphi$. In particular, $\Gamma_{4+2, a}^{-}$does not vanish (whereas $\Gamma_{4+2, D}^{-}=0$ ) and it receives contributions from operators $A_{k}^{-}$with $k \geq 14$ (operators with $Z$ or $\varphi$ ).

The effective action functional for Dirac neutrinos has a number of interesting regularities. They are more clearly exposed by writing $\Gamma_{4+2, D}^{+}$explicitly as done in eqs. (10.1-2) of [51] (for quarks). First, there is just one coupling, $a_{1}$, for all the terms. More interestingly, $\varphi$ and $Z$ appear solely in the form $\operatorname{Im} F[\varphi+Z]$ (recall that $(\varphi+Z)^{*}=(\varphi-Z)$ in Euclidean space). This suggests some kind of analytical dependence since clearly, this is not the most general possible dependence of a functional on the two variables $\varphi$ and $Z$. A well defined pattern of dependence on $Z \pm \varphi$ was identified at the level of $K_{\text {Dirac }}$ in (5.13), as well as in $K_{1}$ in (5.10) for Majorana neutrinos, but the implications are not obvious since the number of $\varphi$ 's is not preserved by subsequent manipulations.

\footnotetext{
${ }^{8}$ Ref. [50] reported a non null coupling to $A_{21}^{-}$for quarks, which would result in a similar coupling for Dirac neutrinos, however that coupling has been shown to vanish in [51-53]. The calculations in [51, 52] are based on eq. (2.22), first derived in [61], and so their method differs from that used in the calculation of [50]. On the other hand the calculations of $[47,50,53]$ follow the method given in [71] which uses the current to reconstruct the effective action.
} 
It is not clear why or how, a (complex) variation in $\varphi$ could be canceled by doing a similar variation in the $Z$. We have been unable to verify whether this "symmetry" persists in other terms of the effective action (not necessarily of the type $4+2$ and CP odd), and it is possible that this is just an accidental symmetry due to the low order of the terms considered. Indeed, at the level of $4+2$ not many terms can be written violating the structure $\operatorname{Im} F[\varphi+Z]$, essentially only those of the form $W^{+} W^{+} W^{-} W^{-}(\varphi+Z)(\varphi-Z)$.

We have investigated whether the structure $\Gamma \sim \operatorname{Im} F[\varphi+Z]$ also shows up in the effective action of the Majorana neutrinos, $\Gamma_{4, M}$. From the results shown in the tables, we find that the symmetry persists in $\Gamma_{4+2, a}^{+}$and $\Gamma_{4+2, b}^{-}$. In $\Gamma_{4+2, b}^{+}$it is broken by a term $i 2\left(b_{1}+b_{2}\right)\left(A_{38}^{+}-A_{36}^{+}\right)$, and in $\Gamma_{4+2, a}^{-}$it is broken by a term $i(8 / 3) a_{2} A_{25}^{-}$. Nevertheless, to reach a firm conclusion it would be necessary to lift the simplifying assumption $\phi(\partial I / \partial \phi)=$ $-2 I$ (see (6.16)) in the integration by parts, which affects the dependence on $\varphi_{\mu}$.

Another regularity found in $\Gamma_{4+2, D}^{+}$is that it has the structure

$$
\begin{aligned}
\Gamma_{4+2, D}^{+} \sim & F_{0}\left[W^{+} W^{+} \hat{D} W^{-} \hat{D} W^{-}\right]+F_{1}\left[W^{+} W^{+} W^{-} \hat{D} W^{-}(\varphi+Z)\right] \\
& +F_{2}\left[W^{+} W^{+} W^{-} W^{-}(\varphi+Z)(\varphi+Z)\right]+\text { c.c. }
\end{aligned}
$$

The position of the derivatives is also not the most general one, even after integration by parts. This suggests that the $(\varphi+Z)$ dependence could be recovered from $F_{0}$ by some kind of gauging, $\hat{D} \rightarrow \hat{D}+\varphi+Z$, but we have been unable to establish whether such a gauging exists.

\section{Invariants and couplings}

In this section we analyze the dependence on $U$ and on the lepton masses of the results just obtained and focus on the couplings of two concrete paradigmatic cases. Throughout this section the diagonal mass matrices of charged leptons and neutrinos are denoted $\hat{m}_{e}$ and $\hat{m}_{\nu}$, reserving $m_{e}$ for the electron mass. Also, no factors of $\phi / v$ are implicit.

\section{$7.1 \quad$ Invariants}

The momentum integrals $I_{a}$ and $I_{b}$ contain two different structures in flavor space

$$
\begin{aligned}
I_{a} & \sim \operatorname{Im} \operatorname{tr}\left(U^{\dagger} f_{1}\left(\hat{m}_{e}\right) U f_{2}\left(\hat{m}_{\nu}\right) U^{\dagger} f_{3}\left(\hat{m}_{e}\right) U f_{4}\left(\hat{m}_{\nu}\right)\right), \\
I_{b} & \sim \operatorname{Im} \operatorname{tr}\left(U^{\dagger} f_{1}\left(\hat{m}_{e}\right) U f_{2}\left(\hat{m}_{\nu}\right) U^{T} f_{3}\left(\hat{m}_{e}\right) U^{*} f_{4}\left(\hat{m}_{\nu}\right)\right) .
\end{aligned}
$$

The first structure is common to Dirac and Majorana cases while the second structure is specific for Majorana neutrinos. By expanding in matrix elements, the $U$-dependent tensors relevant for $I_{a}$ and $I_{b}$ are found to be, respectively,

$$
J_{\alpha \beta}^{i j} \equiv \operatorname{Im}\left(Z_{\alpha}^{i j} Z_{\beta}^{j i}\right), \quad K_{\alpha \beta}^{i j} \equiv \operatorname{Im}\left(Z_{\alpha}^{i j} Z_{\beta}^{i j}\right),
$$

where

$$
Z_{\alpha}^{i j} \equiv U_{\alpha i} U_{\alpha j}^{*}=Z_{\alpha}^{j i *}
$$


Following the standard practice, the labels $\alpha, \beta, \gamma$, etc, refer to the charged leptons and $i, j, k$, etc, to the neutrinos with well-defined masses [36, 59]. All the algebraic properties of the tensors $J$ and $K$ stem from the fact that $U$ is unitary, and this information can be codified in $Z$ under the conditions that, as a matrix with respect to $i j$, i) $Z_{\alpha}=Z_{\alpha}^{\dagger}$, ii) $Z_{\alpha} Z_{\beta}=\delta_{\alpha \beta} Z_{\alpha}$, and $\operatorname{tr}\left(Z_{\alpha}\right)=1$ (i.e., the three $Z_{\alpha}$ are orthogonal projectors on onedimensional subspaces of $\mathbb{C}^{3}$ ). Unfortunately these necessary and sufficient conditions are not linear.

The tensor $J$ is antisymmetric with respect to $i j$ and to $\alpha \beta$ and in fact, for $g=3$, it has only one independent component, the well-known Jarlskog invariant [48]

$$
J_{\alpha \beta}^{i j}=J_{\mathrm{CP}} \quad \text { for }(i j k) \text { and }(\alpha \beta \gamma) \text { cyclic. }
$$

Because $Z_{\alpha}^{i j}$ is invariant under phase redefinitions of the charged leptons, $U_{\alpha i} \rightarrow e^{i \varphi_{\alpha}} U_{\alpha i}$, so are $J_{\alpha \beta}^{i j}$ and $K_{\alpha \beta}^{i j}$. The symmetry of $J_{\alpha \beta}^{i j}$ is larger since it is also invariant under phase redefinitions of the neutrino fields, $U_{\alpha i} \rightarrow U_{\alpha i} e^{i \varphi_{i}}$. This is also the situation for quarks.

Remarkably, although the charged leptons are Dirac fermions as the quarks, the matrix specifically relevant for Majorana neutrinos, $K_{\alpha \beta}^{i j}$, is still antisymmetric in the neutrino sector, $i j$, but symmetric in the charged lepton sector, $\alpha \beta$,

$$
K_{\alpha \beta}^{i j}=-K_{\alpha \beta}^{j i}=K_{\beta \alpha}^{i j} .
$$

In principle this reduces the independent components in $K$ from 81 to 18 (for $g=3$ ). However, the property $\sum_{\alpha} Z_{\alpha}^{i j}=\delta_{i j}$, implies the further 9 conditions

$$
\sum_{\alpha} K_{\alpha \beta}^{i j}=0
$$

which leaves just 9 linearly independent components in $K_{\alpha \beta}^{i j}$ for $g=3$. There are no further linear constraints.

For two generations $J_{\alpha \beta}^{i j}$ vanishes identically (hence the need of at least three flavors to break CP [32]) but $K_{\alpha \beta}^{i j}$ has still one non null component. As a consequence CP violation is allowed in the two generations version of the Standard Model minimally extended to include Majorana neutrinos [59]. We have verified that this is actually the case in our calculation, i.e., there are no accidental cancellations, and so for instance, the coupling to $A_{10}^{-}$is not zero for generic $2 \times 2$ unitary $U$ and generic lepton masses.

Coming back to three generations, the tensor $K_{\alpha \beta}^{i j}$, being antisymmetric in $i j$, can be arranged into three symmetric matrices with respect to $\alpha \beta$, a matrix for each cyclic $(i j)$. Moreover, the sum by columns or by rows in these matrices vanishes and this allows to use the cyclic $(\alpha \beta)$ components to parameterize them:

$$
K^{i j}=\left(\begin{array}{ccc}
-K_{\mu}^{k}-K_{\tau}^{k} & K_{\tau}^{k} & K_{\mu}^{k} \\
K_{\tau}^{k} & -K_{e}^{k}-K_{\tau}^{k} & K_{e}^{k} \\
K_{\mu}^{k} & K_{e}^{k} & -K_{e}^{k}-K_{\mu}^{k}
\end{array}\right), K_{\gamma}^{k} \equiv K_{\alpha \beta}^{i j}(\operatorname{cyclic}(i j k) \text { and }(\alpha \beta \gamma)) \text {. }
$$

(For three generations the diagonal matrix elements $K_{\alpha \alpha}^{i j}$ also serve as independent parameters.) 
The invariance under phase redefinitions of the charged leptons removes (renders ineffective) three out of the nine parameters in $U$, leaving only 6 effective parameters in $K_{\alpha \beta}^{i j}$, namely, 3 angles, one Dirac phase and two Majorana phases. (We have verified that the 6 parameters are truly effective, i.e., the nine $K_{\gamma}^{k}$ fill a six-dimensional submanifold of $\mathbb{R}^{9}$ as $U$ moves in $\mathrm{SU}(3)$.) This implies that not all the nine linearly independent components $K_{\gamma}^{k}$ are truly algebraically independent.

In order to find new constraints, we note that, for any $(i j)$, the determinant of the matrix $K^{i j}$ vanishes while the determinants of the three $2 \times 2$ submatrices are all equal. What is not so trivial is that these determinants are actually independent of the label $(\mathrm{ij})$. Indeed,

$$
K_{\alpha \alpha}^{i j} K_{\beta \beta}^{i j}-K_{\alpha \beta}^{i j} K_{\beta \alpha}^{i j}=J_{\alpha \beta}^{i j} J_{\beta \alpha}^{i j}=-J_{\mathrm{CP}}^{2} \quad \text { for } i \neq j \text { and } \alpha \neq \beta .
$$

The first equality follows just from the definitions of the tensors $K$ and $J$ in terms of $Z$ in (7.1), while the second equality requires $i \neq j$ and $\alpha \neq \beta$ and relies on the fact that there is just a single independent component in $J_{\alpha \beta}^{i j}$. More explictly,

$$
K_{e}^{k} K_{\mu}^{k}+K_{\mu}^{k} K_{\tau}^{k}+K_{\tau}^{k} K_{e}^{k}+J_{\mathrm{CP}}^{2}=0, \quad k=1,2,3 .
$$

This relation implies that the nine invariants $K_{\gamma}^{k}$ can be expressed in terms of six of them plus the Jarlskog invariant, or equivalently, in terms of seven of them. The number of independent parameters is six. This suggests that there exist a further non linear relation among the nine invariants, presumably of polynomial type, but we have not found it.

It is interesting that a $2 \times 2$ symmetric submatrix can be identified with a bidimensional metric and so with a ellipse (the three parameters being the two principal lengths and one rotation angle). Each of the three $K^{i j}$ is equivalent to one such ellipse, and the identity in (7.9) implies that they have the same area. It can be speculated that the missing constraint is related to some other geometrical property of these figures.

\subsection{Couplings of Dirac type}

In order to analyze the couplings obtained, as regards to $\mathrm{CP}$ violation, we consider two cases, one of type $I_{a}$, common to Dirac and Majorana neutrinos, and another of type $I_{b}$ for Majorana neutrinos.

For Dirac neutrinos, all the couplings are proportional to $a_{1}$ so we consider this case. The same coupling appears also for Majorana neutrinos.

$$
a_{1}=I_{a, 1,1,2,2}^{6}=\operatorname{Im} \int \frac{d^{4} p}{(2 \pi)^{4}} p^{6} \operatorname{tr}\left(U^{\dagger} \hat{N}_{e} U \hat{N}_{\nu} U^{\dagger} \hat{N}_{e}^{2} U \hat{N}_{\nu}^{2}\right)
$$

where the propagators $\hat{N}_{e, \nu}$ are diagonal matrices

$$
\hat{N}_{e}=\left(p^{2}+\hat{m}_{e}^{2}\right)^{-1}, \quad \hat{N}_{\nu}=\left(p^{2}+\hat{m}_{\nu}^{2}\right)^{-1} .
$$

This integral is identical to that for quarks in [51], so the results can be taken from there in a direct way:

$$
a_{1}=J_{\mathrm{CP}} \Delta_{\nu} \Delta_{e} I_{\nu e}
$$


where

$$
\begin{aligned}
\Delta_{\nu} & =\left(m_{\nu, 1}^{2}-m_{\nu, 2}^{2}\right)\left(m_{\nu, 2}^{2}-m_{\nu, 3}^{2}\right)\left(m_{\nu, 3}^{2}-m_{\nu 1}^{2}\right), \\
\Delta_{e} & =\left(m_{e}^{2}-m_{\mu}^{2}\right)\left(m_{\mu}^{2}-m_{\tau}^{2}\right)\left(m_{\tau}^{2}-m_{e}^{2}\right), \\
I_{\nu e} & =\int \frac{d^{4} p}{(2 \pi)^{4}} p^{6} \prod_{i=1}^{3} N_{\nu, i}^{2} \prod_{\alpha=1}^{3} N_{e, \alpha}^{2} .
\end{aligned}
$$

At this point approximations can be taken exploiting the big difference between the mass scales of neutrinos and charged leptons. For a generic momentum integral with heavy and light masses

$$
I_{l h}=\int \frac{d^{4} p}{(2 \pi)^{4}} p^{n-4} \prod_{l} N_{l}^{n_{l}} \prod_{h} N_{h}^{n_{h}} \quad\left(0<n<2 \sum_{l} n_{l}+2 \sum_{h} n_{h}\right)
$$

the effective integration range of the variable $p$ is fixed by the light masses and the momentum can be neglected in the heavy propagators, provided the remaining integral is still UV convergent,

$$
\begin{aligned}
I_{l h} & =\frac{1}{\prod_{h} m_{h}^{2 n_{h}}} I_{l} \times\left(1+O\left(\bar{m}_{l}^{2} / \bar{m}_{h}^{2}\right)\right), \\
I_{l} & =\int \frac{d^{4} p}{(2 \pi)^{4}} p^{n-4} \prod_{l} N_{l}^{n_{l}} \quad\left(n<2 \sum_{l} n_{l}\right) .
\end{aligned}
$$

For the coupling $a_{1}$ this implies

$$
a_{1} \approx J_{\mathrm{CP}} \hat{\Delta}_{e} \Delta_{\nu} I_{\nu}
$$

where

$$
\begin{aligned}
\hat{\Delta}_{e} & =\left(\frac{1}{m_{\mu}^{2}}-\frac{1}{m_{e}^{2}}\right)\left(\frac{1}{m_{\tau}^{2}}-\frac{1}{m_{\mu}^{2}}\right)\left(\frac{1}{m_{e}^{2}}-\frac{1}{m_{\tau}^{2}}\right) \approx \frac{1}{m_{e}^{4} m_{\mu}^{2}}, \\
I_{\nu} & =\int \frac{d^{4} p}{(2 \pi)^{4}} p^{6} N_{\nu, 1}^{2} N_{\nu, 2}^{2} N_{\nu, 3}^{2}
\end{aligned}
$$

The experimental value of the leptonic invariant $J_{\mathrm{CP}}$ is not yet well determined, since the value of the Dirac phase is not known. From its definition $\left|J_{\mathrm{CP}}\right| \leq 1 /(6 \sqrt{3})=0.096$ and current data on the angles imply $\left|J_{\mathrm{CP}}\right|<0.039$ [36].

The correct hierarchy of masses, namely, $m_{\nu, 1}<m_{\nu, 3}$ (normal hierarchy) or $m_{\nu, 1}>$ $m_{\nu, 3}$ (inverted hierarchy) is not yet known. ${ }^{9}$ The data on differences between square masses are currently becoming rather precise from several neutrino oscillation experiments [37-40]. With the usual notation, $\Delta m_{i j}^{2}=m_{\nu, i}^{2}-m_{\nu, j}^{2}$, the data indicate that $\Delta m_{21}^{2} \ll\left|\Delta m_{31}^{2}\right|$, and so

$$
\Delta_{\nu} \approx \Delta m_{21}^{2}\left|\Delta m_{31}^{2}\right|^{2}
$$

Specifically, $\Delta m_{21}^{2}=(8.7 \pm 0.2 \mathrm{meV})^{2}$ and $\left|\Delta m_{31}^{2}\right|=(49 \pm 1 \mathrm{meV})^{2}[36] .{ }^{10}$

\footnotetext{
${ }^{9}$ We adopt the standard choice for labeling the neutrinos, namely, $m_{\nu, 1}<m_{\nu, 2}$, and $m_{\nu, 2}-m_{\nu, 1}<$ $\min \left(\left|m_{\nu, 3}-m_{\nu, 1}\right|,\left|m_{\nu, 3}-m_{\nu, 2}\right|\right)$.

${ }^{10}$ Note that $\left|\Delta m_{i j}\right|^{2} \leq\left|\Delta m_{i j}^{2}\right|$, the equal sign requiring a massless neutrino.
} 
On the other hand, there is no precise information on the absolute values of the masses, although the situation is rapidly changing for upper bounds on the sum of neutrino masses, $m_{\nu, 1}+m_{\nu, 2}+m_{\nu, 3}$, from astrophysics data analyzed using available cosmological models $[82,83]$. These bounds are in the range $(0.3-1.3) \mathrm{eV}$, depending on the data and model used [36].

Rather than computing $I_{\nu}$ for generic values of the masses, we will consider three typical scenarios.

a) Quasi degenerate. $m_{\nu, i} \approx \bar{m}_{\nu} \gg\left|\Delta m_{31}^{2}\right|^{1 / 2}\left(\bar{m}_{\nu}\right.$ is a fraction of eV). Using the relation

$$
\int \frac{d^{4} p}{(2 \pi)^{4}} \frac{p^{2 n-4}}{\left(p^{2}+m^{2}\right)^{s}}=\frac{1}{(4 \pi)^{2}} \frac{\Gamma(n) \Gamma(s-n)}{\Gamma(s)} \frac{1}{m^{2(s-n)}} \quad(0<n<s),
$$

one obtains in this case

$$
\Delta_{\nu} I_{\nu} \approx \frac{1}{5} \frac{1}{(4 \pi)^{2}} \frac{1}{\bar{m}_{\nu}^{2}} \Delta m_{21}^{2}\left|\Delta m_{31}^{2}\right|^{2}
$$

b) Normal hierarchy. $m_{\nu, 1} \ll m_{\nu, 2} \approx\left(\Delta m_{21}^{2}\right)^{1 / 2}$, and $m_{\nu, 3} \approx\left|\Delta m_{31}^{2}\right|^{1 / 2}$. In this case the integral $I_{\nu}$ is of the type in (7.14), with $m_{\nu, 1}$ and $m_{\nu, 2}$ as the light masses and $m_{\nu, 3}$ as the heavy one. An estimate of $I_{l h}$ can be obtained by neglecting the light masses in the propagator, provided the remaining integral is still IR convergent,

$$
\begin{aligned}
I_{l h} & =I_{h} \times\left(1+O\left(\bar{m}_{l}^{2} / \bar{m}_{h}^{2}\right)\right), \\
I_{h} & =\int \frac{d^{4} p}{(2 \pi)^{4}} p^{n-4-2 \sum_{l} n_{l}} \prod_{h} N_{h}^{n_{h}} \quad\left(n>2 \sum_{l} n_{l}\right) .
\end{aligned}
$$

In our case (using (7.19))

$$
\Delta_{\nu} I_{\nu} \approx \frac{1}{(4 \pi)^{2}} \Delta m_{21}^{2}\left|\Delta m_{31}^{2}\right|
$$

c) Inverted hierarchy. $m_{\nu, 3} \ll m_{\nu, 1} \approx m_{\nu, 2} \approx\left(\Delta m_{31}^{2}\right)^{1 / 2}$. In this case $m_{\nu, 3}$ is light and $m_{\nu, 1}$ and $m_{\nu, 2}$ are heavy in $I_{\nu}$ and we can apply (7.21). This gives

$$
\Delta_{\nu} I_{\nu} \approx \frac{1}{3} \frac{1}{(4 \pi)^{2}} \Delta m_{21}^{2}\left|\Delta m_{31}^{2}\right|
$$

\subsection{Couplings of Majorana type}

Here we consider the coupling $b_{1}$, typical of $I_{b}$ type,

$$
\begin{aligned}
b_{1} & =I_{b, 1,1,1,2}^{2}=\operatorname{Im} \int \frac{d^{4} p}{(2 \pi)^{4}} p^{2} \operatorname{tr}\left(U^{\dagger} \hat{N}_{e} U \hat{m}_{\nu} \hat{N}_{\nu} U^{T} \hat{N}_{e} U^{*} \hat{m}_{\nu} \hat{N}_{\nu}^{2}\right) \\
& =\sum_{i, j, \alpha, \beta} K_{\alpha \beta}^{i j} m_{\nu, i} m_{\nu, j} \int \frac{d^{4} p}{(2 \pi)^{4}} p^{2} N_{\nu, i} N_{\nu, j}^{2} N_{e, \alpha} N_{e, \beta}
\end{aligned}
$$

At this point, we recall that $K_{\alpha \beta}^{i j}$ contains just nine linearly independent components through antisymmetry in $i j$, and in addition

$$
K_{\gamma \gamma}^{i j}=-K_{\gamma \beta}^{i j}-K_{\gamma \alpha}^{i j}=-K_{\alpha}^{k}-K_{\beta}^{k} \quad(\operatorname{cyclic}(i j k) \text { and }(\alpha \beta \gamma))
$$


Using the identities

$$
\begin{aligned}
N_{\nu, i} N_{\nu, j}^{2}-N_{\nu, j} N_{\nu, i}^{2} & =\left(m_{\nu, i}^{2}-m_{\nu, j}^{2}\right) N_{\nu, i}^{2} N_{\nu, j}^{2}, \\
2 N_{e, \alpha} N_{e, \beta}-N_{e, \alpha}^{2}-N_{e, \beta}^{2} & =-\left(m_{e, \alpha}^{2}-m_{e, \beta}^{2}\right)^{2} N_{e, \alpha}^{2} N_{e, \beta}^{2},
\end{aligned}
$$

the coupling can be written as

$$
b_{1}=-\sum_{k, \gamma} K_{\gamma}^{k} m_{\nu, i} m_{\nu, j}\left(m_{\nu, i}^{2}-m_{\nu, j}^{2}\right)\left(m_{e, \alpha}^{2}-m_{e, \beta}^{2}\right)^{2} \int \frac{d^{4} p}{(2 \pi)^{4}} p^{2} N_{\nu, i}^{2} N_{\nu, j}^{2} N_{e, \alpha}^{2} N_{e, \beta}^{2} .
$$

In this formula $(i j k)$ and $(\alpha \beta \gamma)$ are cyclic permutations of (123) and $(e \mu \tau)$, respectively.

The expression contains nine integrals. For generic masses one could reduce this number to five by adding in each case the missing neutrino or charged lepton propagator squared using the identity $1=N^{2}\left(p^{2}+m^{2}\right)^{2}$. In the case at hand, it is preferable to exploit that the charged leptons are much heavier than the neutrinos to factorize the expression, using (7.15). This gives,

$$
b_{1}=-\sum_{k, \gamma} K_{\gamma}^{k} F_{e, \gamma} F_{\nu, k} \times\left(1+O\left(m_{\nu}^{2} / m_{e}^{2}\right)\right) \approx-\sum_{k} f_{k} F_{\nu, k},
$$

with

$$
\begin{aligned}
& F_{e, \gamma}=\left(\frac{1}{m_{e, \alpha}^{2}}-\frac{1}{m_{e, \beta}^{2}}\right)^{2}, \quad f_{k}=\sum_{\gamma} K_{\gamma}^{k} F_{e, \gamma} \approx \frac{K_{e}^{k}}{m_{\mu}^{4}}+\frac{K_{\mu}^{k}}{m_{e}^{4}}+\frac{K_{\tau}^{k}}{m_{e}^{4}}, \\
& F_{\nu, k}=m_{\nu, i} m_{\nu, j} \Delta m_{i j}^{2} \int \frac{d^{4} p}{(2 \pi)^{4}} p^{2} N_{\nu, i}^{2} N_{\nu, j}^{2} \equiv \frac{1}{(4 \pi)^{2}} \Delta m_{i j}^{2} H\left(m_{\nu, i}^{2} / m_{\nu, j}^{2}\right) .
\end{aligned}
$$

Here we have introduced the dimensionless function

$$
H(x)=(4 \pi)^{2} m_{1} m_{2} \int \frac{d^{4} p}{(2 \pi)^{4}} p^{2} \frac{1}{\left(p^{2}+m_{1}^{2}\right)^{2}} \frac{1}{\left(p^{2}+m_{2}^{2}\right)^{2}}, \quad x=\frac{m_{1}^{2}}{m_{2}^{2}} .
$$

The integral can be conveniently obtained by residues (using e.g. eqs. (11.6,7) of [51])

$$
H(x)=\sqrt{x} \frac{x^{2}-1-2 x \log x}{(x-1)^{3}}, \quad x>0 .
$$

This function increases monotonically from $x=0$ to $x=1$, further

$$
H(x)=H(1 / x), \quad H(x)=\sqrt{x}+O\left(x^{3 / 2} \log x\right), \quad H(1)=\frac{1}{3} .
$$

At present nothing is known about the phases in $U$ and so only bounds can be given on the nine invariants $K_{\gamma}^{k}$. Due to symmetry in the labels, the maximum value of each $\left|K_{\gamma}^{k}\right|$, namely $1 / 4$, is common to all the invariants (but, of course, this extreme is not attained simultaneously for all of them). For instance, for $K_{\mu}^{2}$ it is attained for $\theta_{13}=\pi / 4, \alpha_{13}=\pi / 2$ and $\theta_{12}=\theta_{23}=\alpha_{12}=\delta_{\mathrm{CP}}=0$ (among other sets of values). Here we have used a standard notation for the parameters in $U$ [36]. 
To estimate the effect of the neutrino factor $F_{\nu, k}$ we take the same three scenarios considered previously for Dirac type integrals:

a) Quasi degenerate. If the neutrino masses are similar, $H(1)=1 / 3$ applies and this gives

$$
b_{1} \approx-\frac{1}{3} \frac{1}{(4 \pi)^{2}}\left(\left(f_{1}-f_{3}\right) \Delta m_{21}^{2}+\left(f_{2}-f_{1}\right) \Delta m_{31}^{2}\right) .
$$

b) Normal hierarchy. In this case $m_{\nu, 1} \ll m_{\nu, 2} \ll m_{\nu, 3}$, therefore $H\left(m_{l}^{2} / m_{h}^{2}\right) \approx m_{l} / m_{h}$ applies for the three pairs of neutrino masses, yielding

$$
b_{1} \approx-\frac{1}{(4 \pi)^{2}}\left(-m_{\nu, 2} m_{\nu, 3} f_{1}+m_{\nu, 1} m_{\nu, 3} f_{2}-m_{\nu, 1} m_{\nu, 2} f_{3}\right) .
$$

c) Inverted hierarchy. In this case $m_{\nu, 3} \ll m_{\nu, 1} \approx m_{\nu, 2}$. Now $H\left(m_{\nu, 3}^{2} / m_{\nu, 2}^{2}\right) \approx$ $m_{\nu, 3} / m_{\nu, 2}$ and $H\left(m_{\nu, 1}^{2} / m_{\nu, 2}^{2}\right) \approx 1 / 3$, hence

$$
b_{1} \approx-\frac{1}{(4 \pi)^{2}}\left(m_{\nu, 2} m_{\nu, 3}\left(f_{1}-f_{2}\right)-\frac{1}{3} \Delta m_{21}^{2} f_{3}\right) .
$$

\subsection{Leptons vs quarks and numerical estimates}

It is very instructive to compare the behavior found for leptons with that of quarks. The formulas for quarks are those of type $I_{a}$. Similarly to (7.12), for quarks [51]

$$
g_{\mathrm{CP}}=J_{q} \Delta_{u} \Delta_{d} I_{u d},
$$

however, the integral $I_{u d}$ (the quark analog of $I_{\nu e}$ ) is not so smooth as for leptons. For quarks the relevant splitting between light and heavy is rather $m_{u}, m_{d}, m_{s} \ll m_{c}, m_{b}, m_{t}$ which is superficially similar to $m_{\nu, i} \ll m_{e}, m_{\mu}, m_{\tau}$, but while for the leptons the light particles are all of type "up" (weak isospin $+1 / 2$ ), in the case of quarks the light particles are of mixed up and down type. This is relevant for the coupling due to the structure of the Jarlskog invariant which controls CP violation in the Kobayashi-Maskawa mechanism. After separation of the heavy particles (making use of (7.15))

$$
\Delta_{u} \Delta_{d} I_{u d} \approx \frac{1}{m_{c}^{2}}\left(m_{s}^{2}-m_{d}^{2}\right) I_{q}, \quad I_{q} \equiv \int \frac{d^{4} p}{(2 \pi)^{4}} p^{6} N_{u}^{2} N_{d}^{2} N_{s}^{2} .
$$

As it turns out, as a consequence of IR divergences, the dimensionless quantity $\left(m_{s}^{2}-m_{d}^{2}\right) I_{q}$ is not a continuous function of the masses at $m_{u}=m_{d}=m_{s}=0$. The directional limit there is finite but it depends on how it is taken (i.e., on the ratios $m_{u} / m_{s}$ and $m_{d} / m_{s}$ ). The natural choice $m_{u}, m_{d} \rightarrow 0$ followed by $m_{s} \rightarrow 0$ gives $1 /(4 \pi)^{2}$ which is close to the exact result.

For the Dirac type coupling of leptons,

$$
\Delta_{\nu} \Delta_{e} I_{\nu e} \approx \frac{1}{m_{e}^{4} m_{\mu}^{2}}\left(m_{\nu, 1}^{2}-m_{\nu, 2}^{2}\right)\left(m_{\nu, 2}^{2}-m_{\nu, 3}^{2}\right)\left(m_{\nu, 3}^{2}-m_{\nu 1}^{2}\right) I_{\nu} .
$$

The factor $\left(m_{\nu, 3}^{2}-m_{\nu, 1}^{2}\right) I_{\nu}$ is once again finite but not continuous in the limit $m_{\nu, i} \rightarrow 0$, nevertheless, due to the additional factors $\left(m_{\nu, 1}^{2}-m_{\nu, 2}^{2}\right)\left(m_{\nu, 2}^{2}-m_{\nu, 3}^{2}\right)$, the full expression has a well-defined (zero) limit as $m_{\nu, i} \rightarrow 0$. 


\begin{tabular}{|c|ccc|c|}
\hline & QD & NH & IH & Quarks \\
\hline$\left|a_{1}\right|$ & $7.0 \times 10^{-33}$ & $1.4 \times 10^{-31}$ & $4.8 \times 10^{-32}$ & $3.8 \times 10^{-4}$ \\
$\left|b_{1}\right|$ & $7.4 \times 10^{-11}$ & $2.0 \times 10^{-11}$ & $1.2 \times 10^{-12}$ & $\left(1.2 \times 10^{-7}\right)$ \\
\hline
\end{tabular}

Table 9. Upper bounds (maximal $\left|K_{\gamma}^{k}\right|$ are assumed) for the coefficients of Dirac type $\left(a_{1}\right)$ and Majorana type $\left(b_{1}\right)$ in the three neutrino scenarios: quasi degenerate (QD), normal hierarchy (NH), and inverted hierarchy $(\mathrm{IH})$. The value $\bar{m}_{\nu}=0.1 \mathrm{eV}$ has been adopted in $a_{1}$ for QD neutrinos. For quarks $\left|g_{\mathrm{CP}}\right|$ is shown assuming a maximal value of $J_{q}$. The same coupling using the experimental value of $J_{q}$ is shown below, between parenthesis. Units in $\mathrm{GeV}^{-2}$.

The different behavior of quarks and leptons does not stem from differences in the formulas, but rather from the different pattern of separation between light and heavy particles in each case. In the case of quarks, the IR divergences (a kind of chiral enhancement $[47,50,51,53])$ allows to have a sizable $\mathrm{CP}$ violating effective action even for relatively small quark masses. This idea was forwarded in [47] and first confirmed explicitly in [51].

The previous analysis does not directly extend to the couplings of Majorana type, but we can see that also in this case the amount of $\mathrm{CP}$ violation in the effective action induced by leptons is small as it depends on the small neutrino masses. At least for the lowest dimensional operators, which are those in $\Gamma_{4+2}$. Nevertheless, this conclusion could change for the couplings of higher dimensional operators. As the dimension of the operator increases, the integrals become more UV convergent, but also more IR divergent in the massless limit, and so more sensitive to the IR regime.

Since the precise values of the neutrino masses and the PMNS matrix, including phases, are not known yet, the couplings $a_{1}$ and $b_{1}$ cannot be given definite values. In order to have a feeling of the strength of CP violation induced by leptons, we present in table 9 estimates of $a_{1}$ (Dirac type) and $b_{1}$ (Majorana type). These estimates are really upper bounds obtained by taking $\left|K_{\gamma}^{k}\right|=1 / 4$ and $J_{\mathrm{CP}}=1 /(6 \sqrt{3})$, as well as $\bar{m}_{\nu}=0.1 \mathrm{eV}$. For comparison the value of $g_{\mathrm{CP}}$ for quarks is also given, with $J_{q}=1 /(6 \sqrt{3})$. The number in parenthesis is $g_{\mathrm{CP}}$ using the experimental value $J_{q}=3 \times 10^{-5}$ [36]. In the Majorana case, all $\left|f_{k}\right|$ are estimated (or rather bounded) as $\bar{f} \approx 1 /\left(2 m_{e}^{4}\right)$ and $\left|f_{i}-f_{j}\right| \approx 2 \bar{f}$.

The results shown in table 9 indicate that couplings of the type $I_{a}$ are much smaller than those of quarks. It follows that the leptonic corrections to the couplings of operators which already have a quark contribution is extremely small. Note though that even these small values are many orders of magnitude larger that the perturbative estimates discussed in the Introduction, when these are applied to leptons (these give ratios as small as $10^{-93}$.)

The typical values of couplings of Majorana type are about 20 orders of magnitude larger than those of Dirac type. This indicates that $\mathrm{CP}$ violation could serve as a test to discriminate the two types of neutrino masses, provided the $\mathrm{CP}$ violating phases in the PMNS matrix are not too small. The Majorana couplings are smaller that those of quarks but these new couplings are the only ones present for parity odd operators.

Before finishing this section we want to briefly discuss the structure of the other couplings, $a_{2}, b_{2}, b_{3}, b_{4}$ and $b_{5}$ defined in (6.22). 
The relation similar to (7.12) for $a_{2}$ is

$$
a_{2}=I_{a, 1,1,2,3}^{8}=J_{\mathrm{CP}} \Delta_{\nu} \Delta_{e} I_{\nu e}^{\prime},
$$

with

$$
I_{\nu e}^{\prime}=\int \frac{d^{4} p}{(2 \pi)^{4}} p^{8} \sum_{j=1}^{3} N_{\nu, j} \prod_{i=1}^{3} N_{\nu, i}^{2} \prod_{\alpha=1}^{3} N_{e, \alpha}^{2} .
$$

This quantity can be analyzed along the same lines as $a_{1}$ and similar formulas are obtained (e.g., the factor $1 / 5$ in $(7.20)$ becomes $1 / 2$ for $a_{2}$ ). So this coefficient will be of similar size as $a_{1}$ itself.

Rather than doing a detailed analysis of the coefficients it is instructive to look for potentially large values by studying the possible IR divergences in the integrals.

Consider first the integral $a_{1}=I_{a, 1,1,2,2}^{6}$ for leptons in the limit of small neutrino masses. The integral has many contributions of the type

$$
I_{a, 1,1,2,2}^{6} \sim \int d^{4} p p^{6} N_{e, \alpha} N_{e, \beta}^{2} N_{\nu, i} N_{\nu, j}^{2} \quad \text { (leptons) }
$$

and by choosing different labels of type $\nu$ and $e$, we would want to select the worst cases, i.e. the most IR divergent, ones. However, one can see that this integral is IR finite as the neutrino masses go to zero. The same conclusion follows for $a_{2}=I_{a, 1,1,2,3}^{8}$.

If instead of leptons, the analysis of $I_{a, 1,1,2,2}^{6}$ is carried out for quarks, with the masses of $u, d$ and $s$ going to zero, the worse case is

$$
I_{a, 1,1,2,2}^{6} \sim \int d^{4} p p^{6} N_{c} N_{u}^{2} N_{s} N_{d}^{2} \quad \text { (quarks) }
$$

which is IR divergent at a logarithmic rate. Note that due to antisymmetry of $I_{a}$ with respect to up-like and down-like (or neutrinos and charged leptons) separately (see (4.10)) the two up-like ( $c$ and $u$ above) and the two down-like ( $s$ and $d$ above) must be different. The presence of the IR divergence causes the quarks to give a larger contributions than neutrinos in type $I_{a}$ integrals.

Let us consider now the integrals of type $I_{b}$. In this case, the two neutrino labels should still be different but the charged lepton labels can be equal. For $b_{1}=I_{b, 1,1,1,2}^{2}$ a typical contribution is

$$
I_{b, 1,1,1,2}^{2} \sim m_{\nu, i} m_{\nu, j} \int d^{4} p p^{2} N_{e} N_{e} N_{\nu, i} N_{\nu, j}^{2}
$$

The integral would be logarithmically IR divergent as the two neutrino masses go to zero but this is multiplied by the product of the same masses yielding an $O\left(m_{\nu}^{2}\right)$ final result. The analysis is similar for $b_{2}=I_{b, 1,1,1,3}^{4}$. For $b_{3}=I_{b, 1,1,2,2}^{4}, b_{4}=I_{b, 1,1,3,2}^{6}$ and $b_{5}=I_{b, 2,1,2,2}^{6}$ the integrals themselves are IR finite since there are additional powers of $p^{2}$ but no additional neutrino propagators, as compared to $b_{1}$. Therefore, the other $b_{i}$ couplings are not expected to be larger than $b_{1}$. The typical scale of the couplings of type $b$ is set by the factor $\left|\Delta m_{31}^{2}\right| / m_{e}^{4} \approx 10^{-8} \mathrm{GeV}^{-2}$, while $\left|\Delta m_{31}^{2}\right|^{2} /\left(m_{e}^{4} m_{\mu}^{2}\right) \approx 10^{-27} \mathrm{GeV}^{-2}$ sets the scale in the couplings of type $a$. Compared to this, the scale of $\mathrm{CP}$ violation for quarks is set by the factor $1 / m_{c}^{2} \approx 1 \mathrm{GeV}^{-2}$, in dimension six operators. 


\section{Summary and conclusions}

In this work we have undertaken a direct calculation of the couplings to $\mathrm{CP}$ violating operators as induced by the leptonic loop in the Standard Model, extended to include neutrino masses either of pure Dirac type or pure Majorana type. The study is restricted to leading dimension operators, which have been shown to be of dimension six, although four dimensional $\mathrm{CP}$ odd operators would be obtained in the mixed Dirac-Majorana case (see (4.11)). Our calculation is exhaustive at dimension six. The results are collected in tables 2-8. We have confirmed that $\mathrm{CP}$ would be violated in a two generation scenario with Majorana neutrinos present. Also, we show that $\mathrm{CP}$ odd and $\mathrm{P}$ odd operators are produced at dimension six with Majorana neutrinos, while only $\mathrm{P}$ even operators are produced for Dirac neutrinos.

An interesting result of the present study is the derivation of a Klein-Gordon like operator, the operator $K$ (see (5.23)), which describes the propagation of leptons in the sectors $\left\langle\psi_{R} \bar{\psi}_{L}\right\rangle$ and $\left\langle\psi_{L} \bar{\psi}_{R}\right\rangle$, also in the Majorana case, and which produces the correct effective action in all sectors, $\mathrm{CP}$ odd and $\mathrm{CP}$ even, and beyond the derivative expansion.

Two different mechanisms are present for Majorana neutrinos, one (type $I_{a}$ ) is common to the Dirac neutrino case, and a new one (type $I_{b}$ ) is exclusive of the Majorana case. The two types contribute simultaneously to the same set of CP odd operators. We have shown that type $I_{a}$ contributions are identical to those of the Dirac case when no $Z$ or Higgs are involved, but new terms appear when these fields are present, and in particular, $\mathrm{P}$ can be violated in the $\mathrm{CP}$ odd sector at dimension six. At variance with this, type $I_{b}$ terms allow to break $\mathrm{P}$ at dimension six without requiring Higgs or $Z$ fields.

In the discussion of the new invariants of the PMNS matrix introduced by the presence of Majorana masses, we have been able to identify two quadratic relations among the nine invariants and a relation with the Jarlskog invariant (see (7.9)). Because only six parameters of the PMNS are effective in the $I_{b}$ terms (the three angles, the Dirac phase and the two Majorana phases) it follows that one more non-linear relation is missing among the nine invariants but we have not been able to identify it.

The general expressions of the couplings, which come as momentum integrals have been approximated in two typical cases, by exploiting the small neutrino mass, yielding manageable formulas. Numerically we find that the couplings for Dirac neutrinos, or more generally type $I_{a}$ terms, are many (about fifty or sixty) orders of magnitude larger that perturbative estimates, but still much smaller (by about a factor $10^{-28}$ ) than similar contributions from the quark loop. The reason for this difference is not so much the small mass of the neutrinos but the fact that the pattern of breaking between light and heavy fermions is different in quarks and leptons, as regards to weak isospin: for quarks, the three lightest particles, $u, d$ and $s$, are of up and down mixed type, whereas for leptons the three light neutrinos are of type up, and this is relevant for the Jarlskog determinant and the IR structure of the couplings. For the dimension six operators we have considered, the scale of $\mathrm{CP}$ violation in couplings of type $I_{a}$ (Kobayashi-Maskawa mechanism) is controlled by the factor $\left|\Delta m_{31}^{2}\right|^{2} /\left(m_{e}^{4} m_{\mu}^{2}\right) \approx 10^{-27} \mathrm{GeV}^{-2}$ for leptons and by $1 / m_{c}^{2} \approx 1 \mathrm{GeV}^{-2}$ for quarks. 
The scale of $\mathrm{CP}$ violation for couplings of type $I_{b}$ (virtual lepton-number violation) specific of Majorana neutrinos is set by the factor $\left|\Delta m_{31}^{2}\right| / m_{e}^{4} \approx 10^{-8} \mathrm{GeV}^{-2}$. Numerically, these couplings are smaller than quark-induced terms only by about a factor $10^{-7}$, assuming generic phases in the PMNS matrix. More importantly, some of the operators induced by integration of the Majorana neutrinos are exclusive for this mechanism. In particular, parity violating operators appear at dimension six for Majorana neutrinos which are not present for Dirac neutrinos. In principle, this opens the door to distinguish between Dirac and Majorana neutrinos in precision tests involving $\mathrm{CP}$ violation provided the relevant selection rules, for the appropriate operators, can be enforced.

The main limitation of the calculations of the present type regarding phenomenology is the use of a strict derivative expansion, which implies an expansion around zero fourmomentum for the external fields. The lifting of this restriction is a direction in which the present work could be extended. Other directions include studying the case of mixed Dirac and Majorana neutrino masses, which would allow to consider new operators of lower dimension, the study of higher dimensional operators which have a different IR behavior, and the inclusion of finite temperature effects, relevant for baryogenesis scenarios.

\section{A Covariant and ordinary symbols}

In eq. (6.14) there appear the covariant symbols of several operators. These symbols are multiplicative with respect to $x$ but contain derivatives with respect to $p_{\mu}$. These derivatives will be indicated as $\partial_{\alpha}^{p}=\partial / \partial p_{\alpha}$. To compute the symbols we apply the relations (6.13), using for each field its proper gauge connection, namely, $-q A_{e}$ with $q= \pm 1,0,0$, for $W^{ \pm}, Z$ and $\varphi$, respectively.

In general the covariant symbols are infinite series ordered by the number of derivatives. For $\Gamma_{4+2}$ we need to retain two derivatives, counting $D_{e, \mu}, Z_{\mu}$ and $\varphi_{\mu}$ as first order. The correct number of $W$ 's is already selected in eq. (6.14).

\section{Derivatives and gauge and Higgs fields.}

$$
\begin{aligned}
\bar{W}_{\mu}^{ \pm} & =W_{\mu}^{ \pm}+i W_{\alpha \mu}^{ \pm} \partial_{\alpha}^{p}-\frac{1}{2} W_{\alpha \beta \mu}^{ \pm} \partial_{\alpha}^{p} \partial_{\beta}^{p}+O\left(D^{3}\right), \\
\bar{Z}_{\mu} & =Z_{\mu}+i Z_{\alpha \mu} \partial_{\alpha}^{p}+O\left(D^{3}\right), \\
\bar{\varphi}_{\mu} & =\varphi_{\mu}+i \varphi_{\alpha \mu} \partial_{\alpha}^{p}+O\left(D^{3}\right), \\
\bar{D}_{e, \mu} & =i p_{\mu}+\frac{i}{2} F_{\alpha \mu}^{e} \partial_{\alpha}^{p}+O\left(D^{3}\right), \\
\bar{D}_{e, \mu}^{*} & =i p_{\mu}-\frac{i}{2} F_{\alpha \mu}^{e} \partial_{\alpha}^{p}+O\left(D^{3}\right), \\
\bar{D}_{e \pm, \mu} & =\bar{D}_{e, \mu} \pm\left(\bar{Z}_{\mu}+\bar{\varphi}_{\mu}\right) \\
\bar{D}_{\nu, \mu} & =i p_{\mu} \\
\bar{D}_{\nu \pm, \mu} & =\bar{D}_{\nu, \mu} \pm\left(\bar{Z}_{\mu}-\bar{\varphi}_{\mu}\right) \\
\bar{F}_{\mu \nu}^{e} & =F_{\mu \nu}^{e}+O\left(D^{3}\right) .
\end{aligned}
$$




\section{Mass terms.}

$$
\begin{aligned}
& \bar{m}_{e}^{n}=m_{e}^{n}\left(1+n i \varphi_{\alpha} \partial_{\alpha}^{p}-\frac{1}{2}\left(n \varphi_{\alpha \beta}+n^{2} \varphi_{\alpha} \varphi_{\beta}\right) \partial_{\alpha}^{p} \partial_{\beta}^{p}+O\left(D^{3}\right)\right), \quad n=1,2, \ldots \\
& \bar{m}_{\nu}^{n}=m_{\nu}^{n}\left(1+2 n i \varphi_{\alpha} \partial_{\alpha}^{p}-\frac{1}{2}\left(2 n \varphi_{\alpha \beta}+4 n^{2} \varphi_{\alpha} \varphi_{\beta}\right) \partial_{\alpha}^{p} \partial_{\beta}^{p}+O\left(D^{3}\right)\right),
\end{aligned}
$$

Propagators. There are various propagators, $G_{e}, G_{e}^{(2)}, G_{e}^{*}, G_{\nu}$, and $G_{\nu}^{\prime}$. All of them follow the scheme

$$
\bar{G}=N-N H N+N H N H N+O\left(D^{3}\right)
$$

where $N$ is of order zero and $H$ is at least of order one in derivatives. For the various $N$

$$
\begin{aligned}
& N_{e}=N_{e}^{(2)}=U^{\dagger}\left(m_{e}^{2}+p^{2}\right)^{-1} U \\
& N_{e}^{*}=U^{T}\left(m_{e}^{2}+p^{2}\right)^{-1} U^{*} \\
& N_{\nu}=N_{\nu}^{\prime}=\left(m_{\nu}^{2}+p^{2}\right)^{-1}
\end{aligned}
$$

while for the $H$ :

$$
\begin{aligned}
H_{e} & =\bar{m}_{e}^{2}-\overline{D D}_{e-} \bar{D}_{e}-m_{e}^{2}-p^{2}, \\
H_{e}^{(2)} & =\bar{m}_{e}^{2}-\left(\bar{D}_{e-}+2 \bar{\psi}\right)\left(\bar{D}_{e}+2 \bar{\psi}\right)-m_{e}^{2}-p^{2}, \\
H_{e}^{*} & =\bar{m}_{e}^{2}-\bar{D}_{e}^{*} \bar{D}_{e+}^{*}-m_{e}^{2}-p^{2}, \\
H_{\nu} & =\bar{m}_{\nu}^{2}-\bar{D}_{\nu+} \bar{D}_{\nu-}-m_{\nu}^{2}-p^{2} \\
H_{\nu}^{\prime} & =\bar{m}_{\nu}^{2}-\left(\bar{D}_{\nu-}-2 \bar{\psi}\right)\left(\bar{D}_{\nu+}+2 \bar{\psi}\right)-m_{\nu}^{2}-p^{2} .
\end{aligned}
$$

In the formulas with expressions valid to all orders one has to expand in derivatives dropping terms of $O\left(D^{3}\right)$. E.g.,

$$
\begin{aligned}
H_{\nu}^{\prime}= & m_{\nu}^{2}\left(4 i \varphi_{\alpha} \partial_{\alpha}^{p}-\left(2 \varphi_{\alpha \beta}+8 \varphi_{\alpha} \varphi_{\beta}\right) \partial_{\alpha}^{p} \partial_{\beta}^{p}\right) \\
& -i[\not p, \not \mathbf{Z}+\varphi]+\left[\not p, \gamma_{\mu}\left(Z_{\alpha \mu}+\varphi_{\alpha \mu}\right) \partial_{\alpha}^{p}\right]+(\not{Z}+\phi)^{2}+O\left(D^{3}\right) .
\end{aligned}
$$

Alternatively one can use the method of ordinary symbols $[80,81]$. In this case, the equation similar to $(6.12)$ is

$$
\operatorname{Tr} f(D, M)=\int \frac{d^{d} x d^{d} p}{(2 \pi)^{d}} \operatorname{tr} f(D+i p, M) .
$$

After integration over $p_{\mu}$ all covariant derivatives appear only in the form $\left[D_{\mu},\right]$, but unlike the case of covariant symbols, these explictly covariant combinations have to be obtained by hand (essentially moving the $D$ 's to the right using $D_{\mu} X=X D_{\mu}+\hat{D}_{\mu} X$ ). This makes this method less systematic. It should be noted that the cyclic property of the trace can be freely applied to writing the starting pseudodifferential operator, $f(D, M)$, without changing the UV convergent contributions in the final result, however, in general such freedom is not justified for the different terms in $\operatorname{tr} f(D+i p, M)$.

\section{Acknowledgments}

This work has been supported by Spanish DGI (FIS2011-24149) and Junta de Andalucía grant FQM-225. 
Open Access. This article is distributed under the terms of the Creative Commons Attribution License (CC-BY 4.0), which permits any use, distribution and reproduction in any medium, provided the original author(s) and source are credited.

\section{References}

[1] R.N. Mohapatra et al., Theory of neutrinos: A White paper, Rept. Prog. Phys. 70 (2007) 1757 [hep-ph/0510213] [InSPIRE].

[2] J. Bernabeu, A. De Rujula and C. Jarlskog, Neutrinoless Double Electron Capture as a Tool to Measure the $\nu_{e}$ Mass, Nucl. Phys. B 223 (1983) 15 [InSPIRE].

[3] S.R. Elliott and P. Vogel, Double beta decay, Ann. Rev. Nucl. Part. Sci. 52 (2002) 115 [hep-ph/0202264] [INSPIRE].

[4] J.G. Morfin, J. Nieves and J.T. Sobczyk, Recent Developments in Neutrino/Antineutrino Nucleus Interactions, Adv. High Energy Phys. 2012 (2012) 934597 [arXiv:1209.6586] [INSPIRE].

[5] M.C. Gonzalez-Garcia and M. Maltoni, Phenomenology with Massive Neutrinos, Phys. Rept. 460 (2008) 1 [arXiv:0704.1800] [INSPIRE].

[6] G. Bertone, D. Hooper and J. Silk, Particle dark matter: Evidence, candidates and constraints, Phys. Rept. 405 (2005) 279 [hep-ph/0404175] [INSPIRE].

[7] F. Halzen and D. Hooper, High-energy neutrino astronomy: The Cosmic ray connection, Rept. Prog. Phys. 65 (2002) 1025 [astro-ph/0204527] [INSPIRE].

[8] T. Schwetz, M.A. Tortola and J.W.F. Valle, Three-flavour neutrino oscillation update, New J. Phys. 10 (2008) 113011 [arXiv:0808.2016] [INSPIRE].

[9] J. Schechter and J.W.F. Valle, Neutrino Masses in $\mathrm{SU}(2) \times \mathrm{U}(1)$ Theories, Phys. Rev. D 22 (1980) 2227 [inSPIRE].

[10] J. Schechter and J.W.F. Valle, Neutrino Decay and Spontaneous Violation of Lepton Number, Phys. Rev. D 25 (1982) 774.

[11] KAMIOKANDE-II collaboration, K. Hirata et al., Observation of a Neutrino Burst from the Supernova SN 1987a, Phys. Rev. Lett. 58 (1987) 1490 [INSPIRE].

[12] R.M. Bionta et al., Observation of a Neutrino Burst in Coincidence with Supernova SN 1987a in the Large Magellanic Cloud, Phys. Rev. Lett. 58 (1987) 1494 [INSPIRE].

[13] WMAP collaboration, G. Hinshaw et al., Nine-Year Wilkinson Microwave Anisotropy Probe (WMAP) Observations: Cosmological Parameter Results, Astrophys. J. Suppl. 208 (2013) 19 [arXiv: 1212.5226] [INSPIRE].

[14] D.D. Stancil et al., Demonstration of Communication using Neutrinos, Mod. Phys. Lett. A 27 (2012) 1250077 [arXiv:1203.2847] [INSPIRE].

[15] Z.-z. Xing, Flavor mixing and CP-violation of massive neutrinos, Int. J. Mod. Phys. A 19 (2004) 1 [hep-ph/0307359] [inSPIRE].

[16] A.J. Buras and R. Fleischer, Quark mixing, CP-violation and rare decays after the top quark discovery, Adv. Ser. Direct. High Energy Phys. 15 (1998) 65 [hep-ph/9704376] [InSPIRE].

[17] M. Neubert, B decays and CP-violation, Int. J. Mod. Phys. A 11 (1996) 4173 [hep-ph/9604412] [INSPIRE]. 
[18] Y. Grossman, Y. Nir and R. Rattazzi, CP violation beyond the standard model, Adv. Ser. Direct. High Energy Phys. 15 (1998) 755 [hep-ph/9701231] [INSPIRE].

[19] B. Winstein and L. Wolfenstein, The Search for direct CP-violation, Rev. Mod. Phys. 65 (1993) 1113 [INSPIRE].

[20] E.A. Paschos and U. Turke, Quark Mixing and CP-violation, Phys. Rept. 178 (1989) 145 [INSPIRE].

[21] L. Wolfenstein, Present Status of CP-violation, Ann. Rev. Nucl. Part. Sci. 36 (1986) 137 [INSPIRE].

[22] J.F. Donoghue, B.R. Holstein and G. Valencia, Survey of present and future tests of CP violation, Int. J. Mod. Phys. A 2 (1987) 319 [INSPIRE].

[23] CKMfitTer Group collaboration, J. Charles et al., CP violation and the CKM matrix: assessing the impact of the asymmetric B factories, Eur. Phys. J. C $\mathbf{4 1}$ (2005) 1 [hep-ph/0406184] [INSPIRE].

[24] J.H. Christenson, J.W. Cronin, V.L. Fitch and R. Turlay, Evidence for the 2 pi Decay of the k(2)0 Meson, Phys. Rev. Lett. 13 (1964) 138 [INSPIRE].

[25] NA31 collaboration, H. Burkhardt et al., First Evidence for Direct CP-violation, Phys. Lett. B 206 (1988) 169 [INSPIRE].

[26] BABAR collaboration, B. Aubert et al., Observation of CP-violation in the $B^{0}$ meson system, Phys. Rev. Lett. 87 (2001) 091801 [hep-ex/0107013] [INSPIRE].

[27] A.D. Sakharov, Violation of CP Invariance, c Asymmetry and Baryon Asymmetry of the Universe, Pisma Zh. Eksp. Teor. Fiz. 5 (1967) 32 [InSPIRE].

[28] M. Trodden, Electroweak baryogenesis, Rev. Mod. Phys. 71 (1999) 1463 [hep-ph/9803479] [INSPIRE].

[29] T. Ibrahim and P. Nath, CP Violation From Standard Model to Strings, Rev. Mod. Phys. 80 (2008) 577 [arXiv:0705.2008] [inSPIRE].

[30] M. Pospelov and A. Ritz, Electric dipole moments as probes of new physics, Annals Phys. 318 (2005) 119 [hep-ph/0504231] [INSPIRE].

[31] H.-Y. Cheng, The Strong CP Problem Revisited, Phys. Rept. 158 (1988) 1 [INSPIRE].

[32] M. Kobayashi and T. Maskawa, CP Violation in the Renormalizable Theory of Weak Interaction, Prog. Theor. Phys. 49 (1973) 652 [INSPIRE].

[33] B. Pontecorvo, Mesonium and anti-mesonium, Sov. Phys. JETP 6 (1957) 429 [InSPIRE].

[34] B. Pontecorvo, Neutrino Experiments and the Problem of Conservation of Leptonic Charge, Sov. Phys. JETP 26 (1968) 984 [INSPIRE].

[35] Z. Maki, M. Nakagawa and S. Sakata, Remarks on the unified model of elementary particles, Prog. Theor. Phys. 28 (1962) 870 [INSPIRE].

[36] Particle Data Group collaboration, J. Beringer et al., Review of Particle Physics (RPP), Phys. Rev. D 86 (2012) 010001 [InSPIRE].

[37] RENO collaboration, J.K. Ahn et al., Observation of Reactor Electron Antineutrino Disappearance in the RENO Experiment, Phys. Rev. Lett. 108 (2012) 191802 [arXiv: 1204.0626] [INSPIRE]. 
[38] Double CHOOZ collaboration, Y. Abe et al., Reactor electron antineutrino disappearance in the Double CHOOZ experiment, Phys. Rev. D 86 (2012) 052008 [arXiv:1207.6632] [INSPIRE].

[39] T2K collaboration, K. Abe et al., Evidence of Electron Neutrino Appearance in a Muon Neutrino Beam, Phys. Rev. D 88 (2013) 032002 [arXiv: 1304.0841] [INSPIRE].

[40] DAYA BAY collaboration, F.P. An et al., Improved Measurement of Electron Antineutrino Disappearance at Daya Bay, Chin. Phys. C 37 (2013) 011001 [arXiv:1210.6327] [INSPIRE].

[41] M.E. Shaposhnikov, Structure of the High Temperature Gauge Ground State and Electroweak Production of the Baryon Asymmetry, Nucl. Phys. B 299 (1988) 797 [INSPIRE].

[42] M. Dine, P. Huet, J. Singleton, Robert L. and L. Susskind, Creating the baryon asymmetry at the electroweak phase transition, Phys. Lett. B 257 (1991) 351 [INSPIRE].

[43] X. Zhang and B.L. Young, Effective Lagrangian approach to electroweak baryogenesis: Higgs mass limit and electric dipole moments of fermion, Phys. Rev. D 49 (1994) 563 [hep-ph/9309269] [INSPIRE].

[44] A. Lue, K. Rajagopal and M. Trodden, Semianalytical approaches to local electroweak baryogenesis, Phys. Rev. D 56 (1997) 1250 [hep-ph/9612282] [INSPIRE].

[45] J. García-Bellido, D.Y. Grigoriev, A. Kusenko and M.E. Shaposhnikov, Nonequilibrium electroweak baryogenesis from preheating after inflation, Phys. Rev. D 60 (1999) 123504 [hep-ph/9902449] [INSPIRE].

[46] A. Tranberg and J. Smit, Baryon asymmetry from electroweak tachyonic preheating, JHEP 11 (2003) 016 [hep-ph/0310342] [INSPIRE].

[47] J. Smit, Effective CP-violation in the standard model, JHEP 09 (2004) 067 [hep-ph/0407161] [INSPIRE].

[48] C. Jarlskog, Commutator of the Quark Mass Matrices in the Standard Electroweak Model and a Measure of Maximal CP-violation, Phys. Rev. Lett. 55 (1985) 1039 [INSPIRE].

[49] G.R. Farrar and M.E. Shaposhnikov, Baryon asymmetry of the universe in the minimal Standard Model, Phys. Rev. Lett. 70 (1993) 2833 [Erratum ibid. 71 (1993) 210] [hep-ph/9305274] [INSPIRE].

[50] A. Hernandez, T. Konstandin and M.G. Schmidt, Sizable CP-violation in the Bosonized Standard Model, Nucl. Phys. B 812 (2009) 290 [arXiv:0810.4092] [INSPIRE].

[51] C. Garcia-Recio and L.L. Salcedo, CP violation in the effective action of the Standard Model, JHEP 07 (2009) 015 [arXiv: 0903.5494] [INSPIRE].

[52] T. Brauner, O. Taanila, A. Tranberg and A. Vuorinen, Temperature Dependence of Standard Model CP-violation, Phys. Rev. Lett. 108 (2012) 041601 [arXiv:1110.6818] [INSPIRE].

[53] L.L. Salcedo, Leading order one-loop $C P$ and $P$ violating effective action in the Standard Model, Phys. Lett. B 700 (2011) 331 [arXiv:1102.2400] [InSPIRE].

[54] T. Brauner, O. Taanila, A. Tranberg and A. Vuorinen, Computing the temperature dependence of effective CP-violation in the standard model, JHEP 11 (2012) 076 [arXiv: 1208.5609] [INSPIRE].

[55] A. Tranberg, A. Hernandez, T. Konstandin and M.G. Schmidt, Cold electroweak baryogenesis with Standard Model CP-violation, Phys. Lett. B 690 (2010) 207 [arXiv:0909.4199] [INSPIRE]. 
[56] T. Konstandin and G. Servant, Natural Cold Baryogenesis from Strongly Interacting Electroweak Symmetry Breaking, JCAP 07 (2011) 024 [arXiv:1104.4793] [INSPIRE].

[57] T. Yanagida, Horizontal Symmetry and Masses of Neutrinos, Prog. Theor. Phys. 64 (1980) 1103 [InSPIRE].

[58] M. Gell-Mann, P. Ramond and R. Slansky, Complex Spinors and Unified Theories, Conf. Proc. C 790927 (1979) 315 [arXiv: 1306.4669] [InSPIRE].

[59] T. Morii, C. Lim and S. Mukherjee, The physics of the standard model and beyond, World Scientific, Singapore (2002).

[60] C. Giunti and C.W. Kim, Fundamentals of Neutrino Physics and Astrophysics, Oxford University Press, Oxford U.K. (2007).

[61] L.L. Salcedo, The Invariant factor of the chiral determinant, Eur. Phys. J. C 58 (2008) 423 [arXiv:0807.1696] [INSPIRE].

[62] N.G. Pletnev and A.T. Banin, Covariant technique of derivative expansion of one loop effective action. 1., Phys. Rev. D 60 (1999) 105017 [hep-th/9811031] [INSPIRE].

[63] L.L. Salcedo, The method of covariant symbols in curved space-time, Eur. Phys. J. C 49 (2007) 831 [hep-th/0606071] [INSPIRE].

[64] L. Álvarez-Gaumé and E. Witten, Gravitational Anomalies, Nucl. Phys. B 234 (1984) 269 [INSPIRE].

[65] S.L. Adler, Axial vector vertex in spinor electrodynamics, Phys. Rev. 177 (1969) 2426 [INSPIRE].

[66] J.S. Bell and R. Jackiw, A PCAC puzzle: $\pi_{0} \rightarrow \gamma \gamma$ in the $\sigma$-model, Nuovo Cim. A 60 (1969) 47 [inSPIRE].

[67] W.A. Bardeen, Anomalous Ward identities in spinor field theories, Phys. Rev. 184 (1969) 1848 [InSPIRE].

[68] J. Wess and B. Zumino, Consequences of anomalous Ward identities, Phys. Lett. B 37 (1971) 95 [INSPIRE].

[69] E. Witten, Global Aspects of Current Algebra, Nucl. Phys. B 223 (1983) 422 [InSPIRE].

[70] L.L. Salcedo, Derivative expansion for the effective action of chiral gauge fermions: The Normal parity component, Eur. Phys. J. C 20 (2001) 147 [hep-th/0012166] [INSPIRE].

[71] L.L. Salcedo, Derivative expansion for the effective action of chiral gauge fermions. the abnormal parity component, Eur. Phys. J. C 20 (2001) 161 [hep-th/0012174] [INSPIRE].

[72] L.L. Salcedo, Direct construction of the effective action of chiral gauge fermions in the anomalous sector, Eur. Phys. J. C 60 (2009) 387 [arXiv:0804.2118] [INSPIRE].

[73] J.W. Negele and H. Orland, Frontiers in physics. Vol. 68: Quantum many particle systems, Addison-Wesley, Redwood City U.S.A. (1988).

[74] J.S. Dowker and R. Critchley, Effective Lagrangian and Energy Momentum Tensor in de Sitter Space, Phys. Rev. D 13 (1976) 3224 [InSPIRE].

[75] S.W. Hawking, Zeta Function Regularization of Path Integrals in Curved Space-Time, Commun. Math. Phys. 55 (1977) 133 [InSPIRE].

[76] E. Elizalde, S. Odintsov, A. Romeo, A. Bytsenko and S. Zerbini, Zeta regularization techniques with applications, World Scientific, Singapore (1994). 
[77] R.D. Ball, Chiral Gauge Theory, Phys. Rept. 182 (1989) 1 [InSPIRE].

[78] K. Huang, Quarks, leptons 83 gauge fields, World Scientific, Singapore (1992).

[79] F.J. Moral-Gamez and L.L. Salcedo, Derivative expansion of the heat kernel at finite temperature, Phys. Rev. D 85 (2012) 045019 [arXiv:1110.6300] [INSPIRE].

[80] R.I. Nepomechie, Calculating heat kernels, Phys. Rev. D 31 (1985) 3291 [InSPIRE].

[81] L.L. Salcedo and E. Ruiz Arriola, Wigner transformation for the determinant of Dirac operators, Annals Phys. 250 (1996) 1 [hep-th/9412140] [INSPIRE].

[82] K.N. Abazajian et al., Cosmological and Astrophysical Neutrino Mass Measurements, Astropart. Phys. 35 (2011) 177 [arXiv:1103.5083] [InSPIRE].

[83] Planck collaboration, P.A.R. Ade et al., Planck 2013 results. XVI. Cosmological parameters, Astron. Astrophys. (2014) [arXiv:1303.5076] [INSPIRE]. 